\title{
Current Status of Autophagy Enhancers in Metabolic Disorders and Other Diseases
}

\author{
Kihyoun Park and Myung-Shik Lee*
}

Severance Biomedical Science Institute/Department of Internal Medicine, Yonsei University College of Medicine, Seoul, Korea and Soonchunhyang Institute of Medi-bio Science, Soonchunhyang University, Cheonan, Korea

Autophagy is pivotal in the maintenance of organelle function and intracellular nutrient balance. Besides the role of autophagy in the homeostasis and physiology of the individual tissues and whole organism in vivo, dysregulated autophagy has been incriminated in the pathogenesis of a variety of diseases including metabolic diseases, neurodegenerative diseases, cardiovascular diseases, inflammatory or immunological disorders, cancer and aging. Search for autophagy modulators has been widely conducted to amend dysregulation of autophagy or pharmacologically modulate autophagy in those diseases. Current data support the view that autophagy modulation could be a new modality for treatment of metabolic syndrome associated with lipid overload, human-type diabetes characterized by deposition of islet amyloid or other diseases including neurodegenerative diseases, infection and cardiovascular diseases. While clinically available bona fide autophagy modulators have not been developed yet, it is expected that on-going investigation will lead to the development of authentic autophagy modulators that can be safely administered to patients in the near future and will open a new horizon for treatment of incurable or difficult diseases.

Keywords: autophagy, modulator, metabolic diseases, lysosome, mitochondria, endoplasmic reticulum

\section{INTRODUCTION}

Autophagy, derived from Greek words meaning "self-devouring," is a cellular process of degradation of cell's own material including organelles, proteins or cellular fluid in lysosome acting as the effector organelle of autophagic degradation (Mizushima and Komatsu, 2011; Agudo-Canalejo et al., 2021). Three major types of autophagy such as macroautophagy, microautophagy and chaperone-mediated autophagy have been described (Klionsky and Emr, 2000). Among them, macroautophagy (henceforth referred to as autophagy) is characterized by autophagosome which is encircled by double membrane assembled by subcellular membrane rearrangement sequestering organelles, target substrates and cytoplasm. After autophagosome fusion to lysosome, autophagolysosomes are formed in which sequestered material is decomposed or lysed by lysosomal enzymes (Klionsky and Emr, 2000). The main purpose of autophagy is quality control of organelles or proteins and protection of intracellular homeostasis or nutritional balance during energy deficiency. Thus, when nutrients are deficient, autophagy is initiated to mobilize intracellular nutrients and to avoid harmful effects caused by deficiency of vital nutrients or elements. When organelles are damaged, stressed or dysfunctional, autophagy receptors are recruited and act as bridges linking cargos to LC3 family proteins to initiate autophagic process. Detailed molecular and cellular mechanisms of the

and Other Diseases.

Front. Cell Dev. Biol. 10:811701.

doi: 10.3389/fcell.2022.811701 progression of autophagy after the initiation steps have been extensively investigated, and the 
Nobel Prize for Physiology or Medicine 2016 was bestowed on Yoshinori Ohsumi, as an accolade to his epoch-making discovery of the Atg conjugation system in autophagosome expansion.

Since autophagy is critical in the maintenance of cellular homeostasis and organelle function, autophagy affects diverse aspects of numerous physiological and pathological processes. Hence, dysregulated autophagy would lead to or be associated with a variety of diseases such as metabolic disorders including type 2 diabetes (T2D) or metabolic syndrome, neurodegenerative disorders including Alzheimer's disease, Parkinson's disease or Huntington's disease, immune/inflammatory disorders, infectious diseases and cancer, etc. In T2D or metabolic syndrome, insulin and its downstream mTOR are well-known inhibitors of autophagy, while glucagon, a counter-regulatory hormone of insulin, is one of the first known inducers of autophagy (Deter and de Duve, 1967; Pfeifer, 1978; Sarbassov et al., 2005a). Furthermore, endoplasmic reticulum (ER) and mitochondria that play crucial roles in $\beta$-cell survival or function, insulin sensitivity or resistance and metabolic inflammation or inflammasome activation (Ozcan et al., 2004; Petersen et al., 2004; Laybutt et al., 2007; Misawa et al., 2013), rely on autophagy for proper function. Thus, autophagy is expected to affect diverse aspects of the pathogenesis of T2D or metabolic syndrome.

Because autophagy is involved in such various biological processes and diseases, searches for autophagy modulators have been conducted to develop novel compounds with therapeutic effects against those diseases or aging (Zhang et al., 2007; Eisenberg et al., 2009; Rubinsztein et al., 2012; Shoji-Kawata et al., 2013). In this review, the current development of autophagy modulators for clinical application will be summarized with a focus on therapeutic application to metabolic disorders. Since numerous papers are being published regarding autophagy enhancers for metabolic disorders and other diseases such as neurodegenerative diseases or cardiac disease, apologies are presented to the authors of the work which inadvertently was not covered in this review.

\section{ROLE OF AUTOPHAGY IN THE SYSTEMIC METABOLISM AND METABOLIC DISORDERS}

The role of autophagy in the maintenance of systemic or global metabolic homeostasis and in the pathogenesis of metabolic disorders has been controversial. In vivo role of autophagy in metabolic disorders has been mostly studied using genetic or sitespecific autophagy gene-knockout (KO) mouse models that showed diverse metabolic features depending on the site of genetic alteration (Kim and Lee, 2014). For example, mice with $\mathrm{KO}$ of Atg7, an essential autophagy gene in pancreatic $\beta$ cells producing insulin, showed structural and functional defects of pancreatic $\beta$-cells, resulting in glucose intolerance and susceptibility to diabetes in the presence of metabolic stress (Ebato et al., 2008; Jung et al., 2008; Quan et al., 2012a) (Figure 1). On the contrary, $\mathrm{KO}$ of $\operatorname{Atg} 7$ in insulin target tissues such as skeletal muscle cells or hepatocytes led to the induction of fibroblast growth factor 21 (FGF21) as a "mitokine" due to mitochondrial stress and to the resistance to diet-induced obesity and insulin resistance (Kim K. H. et al., 2013), contrary to the expectation that autophagy deficiency associated with mitochondrial dysfunction in insulin target tissues would lead to insulin resistance. In contrast, increased hepatic accumulation of lipid droplet has also been reported in mice with hepatocytespecific Atg5 KO or Atg7 knockdown (Singh et al., 2009a; Yang et al., 2010) (Figure 1).

In mice with adipose tissue-specific $\mathrm{KO}$ of Atg7, defective differentiation of white adipocytes and reduced fat mass were observed (Singh et al., 2009b; Zhang et al., 2009). In contrast, adipose-specific KO of autophagy genes such as Atg3, Atg16L1 or Beclin 1 during adult period led to mitochondrial dysfunction, hypertrophic adipocytes due to impaired lipolysis, adipocyte death or inflammation in adipose tissue and peripheral insulin resistance or impaired glucose intolerance (Cai et al., 2018; Son et al., 2020) (Figure 1). Mice with deletion of Atg7 in agoutirelated protein (AgRP) neurons producing orexigenic hormones such as AgRP and neuropeptide Y (NPY) showed reduced fat mass and decreased hyperphagic response (Kaushik et al., 2011), while proopiomelanocortin (POMC) neuron-specific Atg7-KO mice showed obese phenotype and aggravated glucose intolerance after high-fat diet (HFD) feeding imposing metabolic stress (Coupe et al., 2012; Kaushik et al., 2012; Quan et al., 2012b) (Figure 1). Mice with macrophage-specific Atg7 KO showed aggravated inflammasome activation in adipose tissue and metabolic deterioration after HFD feeding, which was associated with aggravated mitochondrial dysfunction (Kang et al., 2016; Lee et al., 2016) (Figure 1).

While these genetic models showed diverse metabolic phenotypes depending on the location and severity of autophagy deficiency, metabolic effects of systemic autophagy insufficiency of physiologically relevant degree rather than tissuespecific autophagy gene $\mathrm{KO}$ have been sparsely investigated. We have reported that global Atg7-haploinsufficient mice showed compromised adaptation to metabolic stress and accelerated progression from obesity to diabetes, which was associated with aggravated liver steatosis and augmented inflammasome activation in adipose tissue (Lim et al., 2014). In a similar vein, Atg $4 b$-KO mice with reduced autophagic competence, exhibited aggravated metabolic profile after HFD (Fernández et al., 2017). Mice that are defective in stimulus-induced autophagy due to knock-in mutations in BCL2 phosphorylation sites (T69A, S70A and $\mathrm{S} 84 \mathrm{~A})$ (Bcl2 AAA mice) also showed impaired exercisemediated protection against glucose intolerance after HFD ( $\mathrm{He}$ et al., 2012). On the other hand, overexpression of Atg5 improved metabolic profile of aged mice (Pyo et al., 2013). These results indicate that adequate systemic autophagy is necessary for host adaptation to metabolic stress without which metabolic deterioration might ensue. These results also suggest the possibility that systemic enhancement of autophagic activity may confer beneficial metabolic effects against metabolic stress by enhancing cellular and global adaptive changes to the stress. In contrast, mice with genetic hyperactivation of autophagy through transgenic expression of constitutively active Becnl $\left(B e c n 1^{\mathrm{F} 121 \mathrm{~A}}\right)$ (Rocchi et al., 2017) displayed improved insulin sensitivity due to 


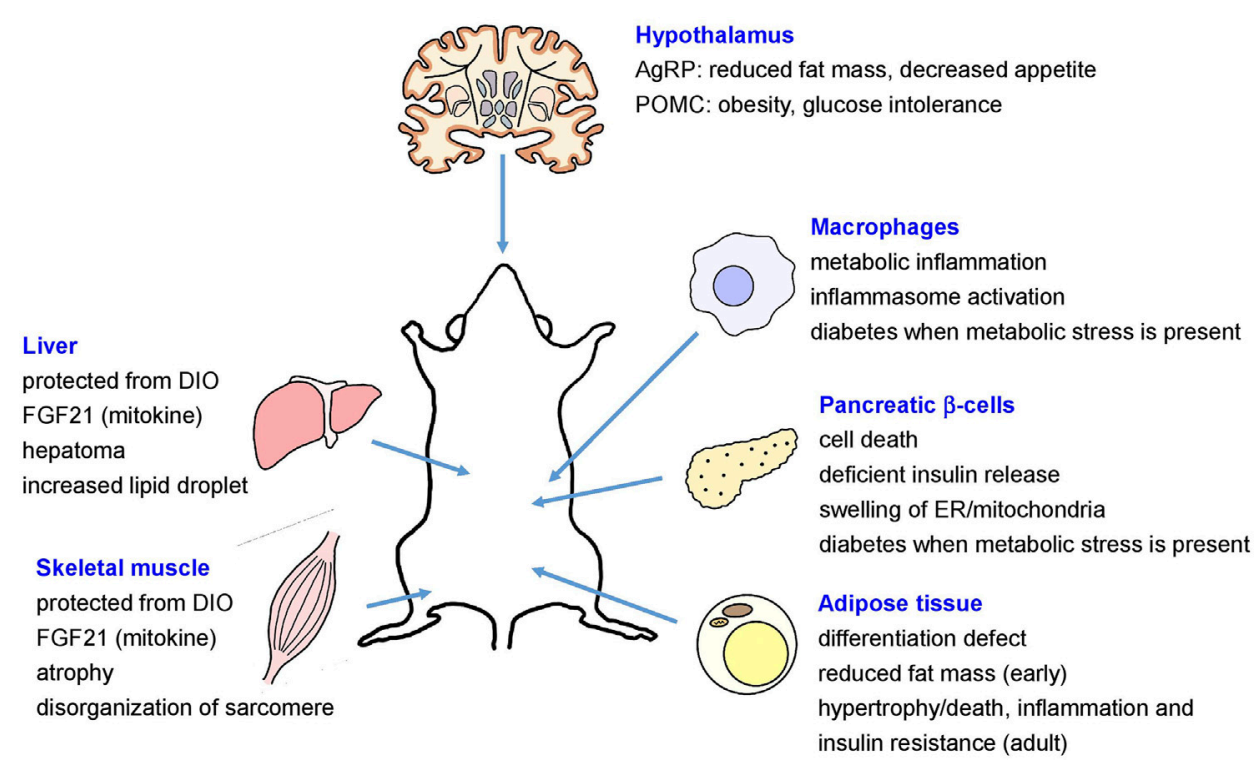

FIGURE 1 | Metabolic effects of site-specific KO of autophagy genes (AgRP, agouti-related protein; DIO, diet-induced obesity; ER, endoplasmic reticulum; FGF21, fibroblast growth factor 21; POMC, proopiomelanocortin).

attenuated endoplasmic reticulum (ER) stress in peripheral tissues but aggravated glucose intolerance due to compromised $\beta$-cell function when fed HFD (Yamamoto et al., 2018). Such results have been ascribed to excessive degradation of insulin secretory granules by hyperactive $\beta$-cell autophagy, termed "vesicophagy," and might suggest that intermittent rather than chronic activation of autophagy of an appropriate degree needs to be considered for treatment of T2D (Yamamoto et al., 2018).

While not an autophagy protein per se, effects of acyl-CoA binding protein (ACBP) involved in transport of acyl-CoA and lipid catabolism on autophagy and systemic metabolism has been studied. Upon autophagy induction, ACBP was released, and extracellular ABCP inhibited autophagy and promoted lipid anabolism or weight gain, which supports the view that systemic autophagy activation promotes lipid catabolism. On the other hand, intracellular ACBP stimulated autophagic flux, suggesting a feedback regulation of autophagy by ACBP, a lipid catabolic factor (Bravo-San Pedro et al., 2019).

\section{AUTOPHAGY IN HUMAN DIABETES OR MURINE DIABETES MIMICKING HUMAN DIABETES}

Most of the studies investigating the role of autophagy in T2D or metabolic disorders have been employing genetic mouse models, and studies of the relationship between autophagy and metabolism in humans are sparse. Technological limitation in the assay of autophagic flux in human tissues or samples could be one of the reasons impeding studies of the role of autophagy in the metabolic regulation in humans. Previous studies of tissue samples from patients with T2D have examined morphological features and number of autophagosomes employing electron microscopy. In pancreatic islets of T2D patients, accumulation of enlarged autophagic vacuoles and autophagosomes was observed which was decreased after treatment with metformin, suggesting impeded removal of autophagic substances in islets of T2D patients (Masini et al., 2009). Protective effects of Imatinibinduced autophagy on human islet cell apoptosis by cytokine combinations have also been reported (Marselli et al., 2013). In human metabolic liver diseases, genetic association between nonalcoholic fatty liver disease (NAFLD) and variants of IRGM, an autophagy gene activated by infection (Grégoire et al., 2011) has been observed (Lin et al., 2016), suggesting the role of decreased autophagy-mediated lipid clearance or lipophagy in the development of human NAFLD.

Although most studies on the relationship between autophagy and metabolic diseases have been conducted using genetic mouse models as mentioned above, murine models of metabolic diseases cannot exactly recapitulate human metabolic diseases. Specifically, pathophysiological features of human diabetes and murine one are different in several aspects. One of the most striking differences is amyloid deposition in pancreatic islets of $>90 \%$ of T2D patients but not in those of murine diabetes, which is due to different amino acid sequences of amylin or islet amyloid polypeptide (IAPP). Human IAPP (hIAPP) is amyloidogenic, while murine IAPP (mIAPP) is non-amyloidogenic because proline-rich sequence in residues 20-29 of mIAPP inhibits $\beta$-sheet formation, a prerequisite for amyloidogenesis (Westermark et al., 2011). It is unknown why hIAPP acquired amyloidogenic propensity during evolution. Because insoluble or amyloid proteins are preferentially cleared by autophagic pathway or lysosomal degradation due to their large size in contrast to soluble or non-amyloid proteins that can be cleared by both proteasomal and autophagic pathways (Rubinsztein, 2006), autophagy may be more important in human diabetes than in murine diabetes. 


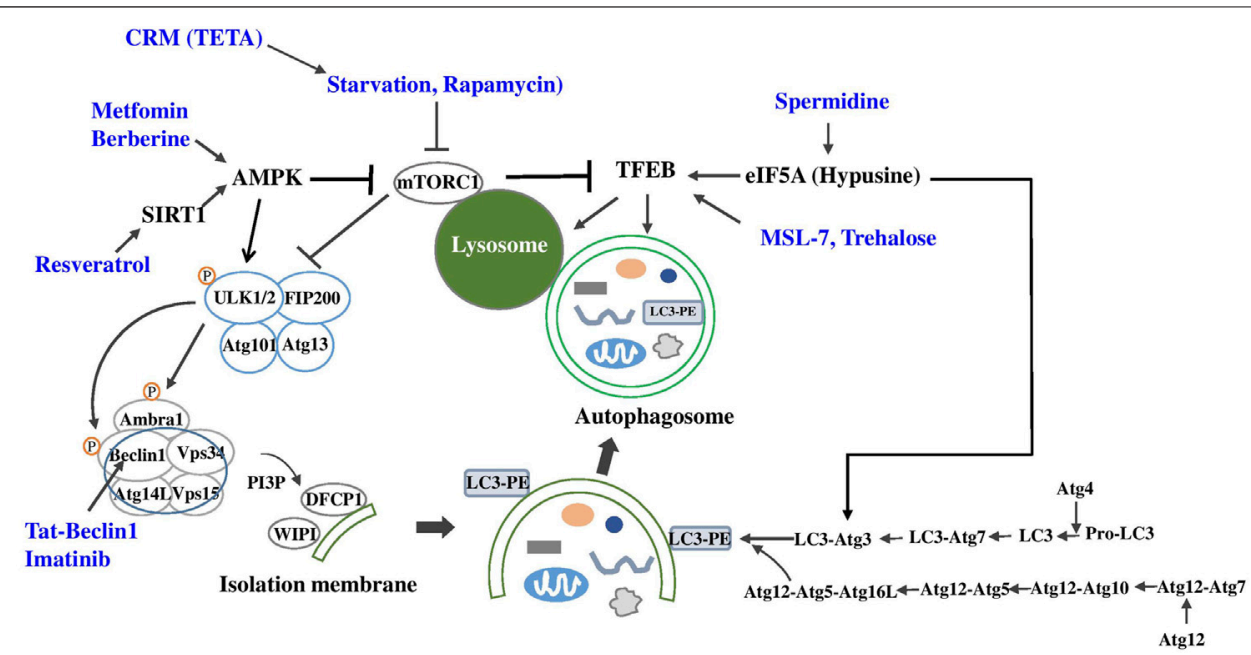

FIGURE 2 | Autophagy enhancers for metabolic diseases (blue) and their target molecules (black) in autophagy machinery (CRM, calorie restriction mimic; TETA, Triethylenetetramine dihydrochloride).

To study the role of autophagy in human-type diabetes characterized by islet amyloid accumulation, we have employed mice expressing $h I A P P$ in pancreatic $\beta$-cells driven by rat insulin promoter ( $h I A P P^{+}$mice). While $h I A P P^{+}$mice developed only mild hyperglycemia, $h I A P P^{+}$mice crossed to $\beta$-cell-specific autophagy-KO mice ( $h I A P P^{+} A \operatorname{tg} 7^{\Delta \beta \text {-cell }}$ mice) developed overt diabetes, accompanied by hIAPP oligomer and amyloid accumulation in pancreatic islets (Kim et al., 2014; Rivera et al., 2014). A study using hIAPP knock-in mice instead of $h I A P P^{+}$mice also showed essentially similar results (Shigihara et al., 2014). These results demonstrate a critical role of $\beta$ cell autophagy in the clearance of hIAPP oligomer and in the prevention of islet amyloid deposition, which has implication in the understanding and treatment of human diabetes.

\section{AUTOPHAGY ENHANCER IN METABOLIC DISORDERS}

As the above results suggested potential therapeutic effects of autophagy modulation in $\mathrm{T} 2 \mathrm{D}$ or metabolic syndrome and human-type diabetes, several autophagy enhancers have been developed or rediscovered from known drugs or chemicals against those diseases (Table 1).

\section{Berberine}

Berberine, a traditional Chinese medicine, has been reported to improve metabolic profile of experimental animals or human patients with metabolic syndrome, diabetes or NAFLD which has been attributed to activation of AMPK and suppression of reactive oxygen species or inflammatory changes (Ma et al., 2018) (Figure 2). Recent papers showed the role of autophagy activation in the metabolic improvement by Berberine which was dependent on SIRT1 activation (Sun et al., 2018; Zheng et al., 2021). Autophagy-independent effects such as FGF21 induction has also been reported to contribute to the metabolic improvement by Berberine (Sun et al., 2018).

\section{Imatinib}

Imatinib is a well-known anti-cancer drug that has a dramatic effect on chronic myelogenous leukemia (CML) and gastrointestinal stromal tumor by specifically inhibiting bcr$\mathrm{Abl}$ and Kit kinases (Druker et al., 1996; Duensing et al., 2004). Imatinib has been reported to enhance autophagic activity of several cancer cells which may be related to the development of resistance against Imatinib. While conclusive mechanism of autophagy induction by Imatinib is still not clear, the mechanism of autophagy induction by Imatinib has been attributed to inhibition of Kit or Abl, increase of PARKIN-Beclin 1 interaction, induction of Xiap, Atg5 or Beclin 1, and ER stress (Bellodi et al., 2009; Can et al., 2013; Etmer et al., 2007; Lin et al., 2015; Xie et al., 2017) (Figure 2). Imatinib can improve metabolic profile of experimental animals or patients with metabolic syndrome or diabetes (Breccia et al., 2005; Veneri et al., 2005; Han et al., 2009), which may be related to autophagy enhancement, while autophagy-independent mechanisms such as reduction of TNF- $a$ release, ER stress or JNK activation might also contribute to the improved metabolic profile by Imatinib (Han et al., 2009). Furthermore, Imatinib could induce remission of diabetes of obese mice with insufficient autophagy, which was accompanied by autophagy induction in vivo and reduced insulin resistance (Lim et al., 2014). When Imatinib is considered as therapeutic agent inducing autophagic activity, potential adverse effects of Imatinib such as peripheral edema, cardiotoxicity and hematological side effects should be considered (Hartmann et al., 2009; Orphanos et al., 2009).

\section{MSL-7}

We recently identified and developed autophagy enhancer small molecules in a screening of a chemical library. We observed that MSL can enhance nuclear translocation of TFEB, a principal regulator of autophagy gene expression and lysosome biogenesis (Settembre et al., 2011) in an mTORC1-independent manner (Lim et al., 2018) (Figure 2). We chemically modified MSL to 
enhance microsomal stability (MSL-7), and MSL-7 was able to exert beneficial metabolic effects on obese mouse models by enhancing the clearance of lipid in the liver tissue and ameliorating metabolic inflammation in adipose tissue (Lim et al., 2018). Attenuation of metabolic inflammation was due to amelioration of fatty acid-induced mitochondrial dysfunction and downregulation of fatty acid-induced inflammasome activation since mitochondrial events are important players in inflammasome activation (Misawa et al., 2013).

While these results showed improvement of metabolic profile of HFD-fed mice through enhanced clearance of lipid and reduced inflammasome activation, another potential metabolic effect of MSL-7 was studied because amyloidogenic hIAPP oligomer or islet amyloid (Westermark et al., 2011), is preferentially cleared by autophagy or lysosomal proteolysis rather than proteasomal degradation (Rubinsztein, 2006; Kim et al., 2014). Indeed, MSL-7 administration was able to improve glucose tolerance and $\beta$-cell function of HFD-fed $h I A P P^{+}$mice expressing amyloidogenic hIAPP in $\beta$-cells, which was accompanied by reduction of hIAPP oligomer, islet amyloid or $\beta$-cell apoptosis and restoration of $\beta$-cell mass or insulinogenic index representing $\beta$-cell function (Kim et al., 2021b). These results suggest the possibility that autophagy enhancer may have more significant effects on human diabetes compared to its effects on murine diabetes because of additional effect of autophagy enhancer on $\beta$-cell viability and function.

\section{Rapamycin}

Rapamycin is a classical autophagy enhancer acting through inhibition of mTOR (Klionsky and Emr, 2000) (Figure 2). mTOR can associate with other factors forming two mTOR complexes, mTORC1 and mTORC2. mTORC1 complex consists of mTOR, Raptor (regulator-associated protein of mTOR), PRAS40 (proline-rich Akt substrate, $40 \mathrm{kDa}$ ), Deptor and mLst8 (mammalian lethal with SEC13 protein 8), while mTORC2 complex comprises mTOR, Rictor, mSin1 (mammalian stress-activated protein kinase-interacting protein 1), Protor $1 / 2$, Deptor and mLst8 (Ardestani et al., 2018). It is generally held that $\mathrm{mTORC} 1$ is sensitive to rapamycin, whereas mTORC2 is not. However, prolonged rapamycin treatment has been shown to inhibit mTORC2 (Sarbessov et al., 2006).

Rapamycin has been administered to several models of diabetes. Rapamycin has been shown to improve metabolic profile and $\beta$-cell function of Akita mice with a mutation in proinsulin gene leading to proinsulin misfolding and severe ER stress, and such improved $\beta$-cell function was attributed to autophagy enhancement (Bachar-Wikstrom et al., 2013). However, prolonged administration of Rapamycin has deleterious effects on $\beta$-cell mass which is due to abrogation of mTORC1 activity positively modulating $\beta$-cell mass (Barlow et al., 2013). Prolonged Rapamycin administration can also impair insulin sensitivity, which could be due to inhibition mTORC2, since mTORC2 is PDK2 regulating Akt473 phosphorylation by insulin (Sarbassov et al., 2005b).

The effect of Rapamycin on longevity has been a controversial topic. Rapamycin was shown to prolong lifespan of mice (Harrison et al., 2009), while the role of autophagy in this model is unclear. In contrast to the effect of Rapamycin on longevity, Rapamycin had no clear effect on aging-related features such as kyphosis, gait disturbance, motor incoordination, impaired hippocampal neurogenesis, decreased grip strength, glomerulosclerosis or decreased $\mathrm{CD}^{+} \mathrm{T}$ cell number, suggesting that Rapamycin may prolong lifespan of mice independent of its effect on aging (Neff et al., 2013). In contrast, anti-aging effect or reversal of aging by Rapamycin such as reversal of reduced hematopoietic stem cell renewal, increased left cardiac ventricular mass and compromised myocardial performance or diastolic function in aged mice, have also been reported (Chen et al., 2009; Dai et al., 2014). In contrast to its effect on non-diabetic mice, Rapamycin shortened lifespan of diabetic $d b / d b$ mice (Sataranatarajan et al., 2016).

\section{Resveratrol}

Resveratrol, which is a natural polyphenol in red wine and wellknown activator of SIRT1, has been reported to improve metabolic profile and extend healthspan of HFD-fed mice, which was associated with increased biogenesis of mitochondria and enhanced activities of PGC-1 $\alpha$ or AMPK (Baur et al., 2006) (Figure 2). Resveratrol can promote autophagic activity through SIRT1-dependent deacetylation of autophagy proteins or TFEB (Morselli et al., 2011; Zheng et al., 2021). However, resveratrol administration for 6 months did not have beneficial metabolic effects in human patients with T2D (Bo et al., 2016). In contrast, small but significant effects of resveratrol on blood glucose, lipid profile or bone mineral density of patients with T2D have recently been reported (Bo et al., 2018; Hoseini et al., 2019).

\section{Rg2}

Rg2, identified in a chemical screening using GFP-LC3 system, has been shown to improve glucose profile, insulin sensitivity and fatty liver change of mice fed HFD through autophagy activation (Fan et al., 2017). Rg2 also reduced body and fat weights of mice fed HFD; however, this effect on body weight was independent of autophagy.

\section{Spermidine}

Spermidine, a polyamine synthesized in vivo from ornithine, has been reported to induce autophagy through epigenetic regulation of autophagy genes. Spermidine inhibits EP300 (E1A-binding protein p300), a lysine acetyltransferase and endogenous inhibitor of autophagy (Pietrocola et al., 2015). Spermidine has been reported to improve metabolic profile and liver steatosis of HFD-fed mice through autophagy activation, which was accompanied by reduced metabolic inflammation, enhanced thermogenesis and improved gut barrier function (Ma et al., 2021). However, autophagy-independent effects such as antioxidant effect or nitric oxide (NO) production might also play a role in the metabolic improvement by spermidine (Madeo et al., 2018).

In addition to the effect of spermidine on metabolic diseases, spermidine may affect aging. Given that spermidine level decreases in multiple organs by aging (Nishimura et al., 2006), decreased autophagic activity in aging (Hansen et al., 2018) may 
be causally related to spermidine insufficiency. Indeed, spermidine could extend longevity of $C$. elegans and Drosophila (Eisenberg et al., 2009). Furthermore, spermidine has also been reported to extend lifespan of aged mice by enhancing autophagy (Eisenberg et al., 2016). In a recent paper, spermidine has been shown to induce hypusination of eIF5A which is required for efficient translation of poly-proline proteins such as TFEB and ATG3, which led to the reversal of immune senescence of B lymphocytes (Zhang H. et al., 2019) and protection against premature brain aging or mitochondrial dysfunction (Liang et al., 2021) (Figure 2). The effect of spermidine on $\mathrm{T}$ cell autophagy has also been reported. Spermidine has been shown to enhance cytokine response of $\mathrm{T}$ cells from old vaccinees with suppressed autophagic activity by maintaining TFEB and autophagy (Alsaleh et al., 2020). It will be intriguing to investigate whether spermidine administration can reverse senescence or aging of other types of cells through similar mechanisms.

\section{Trehalose}

Trehalose is a glucose dimer acting as a chemical chaperone, and has been reported to enhance autophagy. The mechanism of autophagy induction by trehalose has been attributed to inhibition of solute carrier 2A (SLC2A), a glucose transporter called GLUT, by trehalose, leading to activation of $5^{\prime}$ AMPactivated protein kinase (AMPK) and autophagy (DeBosch et al., 2016). However, since SLC2A inhibition was observed at high concentrations of trehalose above $10 \mathrm{mM}$, it is still not clear whether inhibition of glucose transporter can entirely explain autophagy activation by trehalose. Trehalose has also been reported to activate transcription of autophagy genes or transcription factor EB (TFEB), a master regulator of autophagy gene expression and lysosome biogenesis through low-grade lysosomal stress in an mTOR-independent FOXO1dependent manner (Sarkar et al., 2007a; Dehay et al., 2010; Castillo et al., 2013; Jeong et al., 2021) (Figure 2).

Regarding therapeutic potential, trehalose has been shown to improve glucose profile and insulin sensitivity of obese mice with autophagy insufficiency, which was accompanied by amelioration of fatty liver (Lim et al., 2014). The effect of trehalose has also been tested in mice expressing amyloidogenic human-type IAPP fed HFD. In these mice, administration of trehalose improved glucose profile and augmented insulinogenic index representing $\beta$-cell function. Accumulation of hIAPP oligomer and amyloid in pancreatic islets was also attenuated by trehalose (Kim et al., 2014). These results suggest possible therapeutic potential of autophagy enhancers in human-type diabetes.

Trehalose is degraded by intestinal trehalase, which may affect bioavailability of ingested trehalose. Lentztrehalose, a novel analog of trehalose with reduced susceptibility to trehalase, has been produced (Wada et al., 2015). Lactotrehalose, another trehalase-resistant, has been shown to ameliorate fatty liver after high-sucrose diet feeding (Zhang et al., 2020b). Contrary to these reports showing autophagyenhancing effect of trehalose, a previous paper has questioned autophagy enhancing activity of trehalose (Kaizuka et al., 2016).

\section{Triethylenetetramine Dihydrochloride}

TETA, a $\mathrm{Cu}^{2+}$-chelating agent that has been used for treatment of Wilson's disease, has been shown to improve metabolic profile of HFD-fed or $o b / o b$ mice. Such metabolic effects were related not to chelation of $\mathrm{Cu}^{2+}$ but to stabilization and increased activity of spermidine $\mathrm{N}^{1}$-acyltransferase (SAT1) that is also necessary for beneficial effects of spermidine on host metabolism (Castoldi et al., 2020) (Figure 2).

\section{Other Autophagy Enhancers in Metabolic Diseases}

Digoxin; ikarugamycin, a natural marine-derived product; and alexidin dihydrochloride, a synthetic compound, have been identified in a screening for TFEB agonists and have been reported to have beneficial metabolic effects on metabolic syndrome after HFD feeding and to extend lifespan of $C$. elegans (Wang et al., 2017). Dioscin, a natural steroid saponin, has been reported to improve metabolic profile and liver steatosis of obese mice through autophagy upregulation (Liu M. et al., 2015).

\section{Known Drugs Against T2D or Other Metabolic Diseases}

Some of the effects of drugs that are already being used in the clinic against T2D or other metabolic diseases have been ascribed to autophagy enhancement. Such drugs may work through both the originally proposed mechanisms and autophagy modulation, while the proportion of autophagy modulation in the pharmacological effects of the drug could be different from one drug to another.

\section{Metformin}

Metformin is a widely-used anti-diabetic medicine that has been recommended as the first-line drug against diabetes. While metformin has AMPK-independent mechanisms for improvement of metabolic profile (Kalender et al., 2010), most investigators concur with the opinion that metformin activates AMPK (Zhou et al., 2003). Then, metformin would be able to enhance autophagic activity since AMPK activation can upregulate autophagic activity through direct phosphorylation of ULK1 and Beclin 1, key molecules in the initiation of autophagy, or inhibition of mTORC1 (Egan et al., 2011; Howell et al., 2017; Kim J. et al., 2013; Kim et al., 2011) (Figure 2). Autophagy induction by AMPK activation is in line with the concept that autophagy is an adaptive process in response to nutrient deficiency and that AMPK is a sensor of intracellular energy balance. Hence, well-established AMPK activation by metformin suggests the possibility that improvement of metabolic profile by metformin might be related to autophagy induction through AMPK activation. Consistent with this concept, protection of pancreatic $\beta$-cells against lipoapoptosis by metformin was attributed to autophagy activation (Wu et al., 2013; Jiang et al., 2014). Metformin has also been shown to enhance disposal of accumulated autophagic vacuoles in $\beta$-cells (Masini et al., 2009), and to alleviate aging- 
associated inflammation, fatty liver disease or diabetic kidney disease through autophagy induction (Song et al., 2015; Ren et al., 2019; Bharath et al., 2020). Thus, it is likely that anti-diabetic effect of metformin could be partly due to autophagy activation, in addition to autophagy-independent effects such as inhibition of mitochondrial complex I activity or alteration of gut microbiota (Owen et al., 2000; Shin et al., 2014).

\section{GLP-1 Agonist}

GLP-1 receptor agonists such as liraglutide have been reported to enhance autophagy level of insulinoma cells (Chen et al., 2013). While GLP-1 receptor agonists may upregulate autophagy in several tissues including the liver and thereby improve liver steatosis (Sharma et al., 2011; Noyan-Ashraf et al., 2013; Fang et al., 2020), the mechanism of autophagy induction by of GLP-1 receptor or other G-protein-coupled receptors (GPCR) is unclear (Wauson et al., 2014). In this regard, role of increased AMPK or cGMP and reduced mTOR or GSK3 $\beta$ signaling have been suggested (Candeias et al., 2018; Yang et al., 2018). Increases of autophagy by GLP-1 receptor agonists might also be related to the amelioration of ER stress (Yusta et al., 2006).

Additionally, GLP-1 agonists have been reported to have therapeutic effects on diabetic kidney disease (Yamada et al., 2021) through promotion of autophagy.

\section{SGLT2 Inhibitor}

Sodium-glucose transporter 2 (SGLT2) inhibitor is a novel class of anti-diabetic drug acting by increasing urinary loss of glucose and inducing a starvation-like state. SGLT2 inhibitors have been shown to enhance autophagic activity through AMPK activation (Hawley et al., 2016). SGLT2 inhibitors have also been reported to induce autophagy in diabetic heart and kidney tissues, which might be related to cardio- or reno-protective effects of SGLT2 inhibitors (Packer, 2020a; Packer, 2020b).

\section{PPAR- $\gamma$ Agonist}

Peroxisome proliferator-activated receptor $\gamma(\operatorname{PPAR}-\gamma)$ is a transcriptional factor regulating expression of genes involved in adipogenesis and lipid uptake (Spiegelman, 1998). Rosiglitazone, a representative PPAR $-\gamma$ agonist, has been reported to protect $\beta$-cells against lipoapoptosis, which was ascribed to the activation of autophagy through AMPK (Wu et al., 2013). On the other hand, inhibition of autophagy by rosiglitazone has also been reported (Ji et al., 2018; Li et al., 2018).

Pioglitazone, another PPAR- $\gamma$ agonist, has also been reported to ameliorate fatty liver disease through autophagy activation (Hsiao et al., 2017).

\section{PPAR- $\alpha$ Agonist}

PPAR- $\alpha$ is a transcriptional factor which plays a crucial role in the expression of genes involved in fatty acid oxidation, lipid transport and ketosis (Torra et al., 2000). PPAR- $\alpha$ is induced by fasting (Kersten et al., 1999), suggesting its potential relationship with autophagy induction by fasting. Indeed, PPAR- $\alpha$ has been shown to bind promoters of several autophagy genes. Furthermore, GW7647, a PPAR- $\alpha$ agonist, has been reported to reverse feeding-induced autophagy suppression (Lee et al., 2014). Fenofibrate, a classical PPAR- $\alpha$ agonist, has been reported to alleviate HFD-induced kidney injury in a diabetic kidney disease model probably through autophagy activation (Sohn et al., 2017). While not directly related to diabetes or metabolic diseases, fenofibrate has also been reported to suppress cartilage degeneration in vitro through autophagy activation, and use of fenofibrate has been associated with improved clinical course of osteoarthritis in human patients (Nogueira-Recalde et al., 2019).

On the contrary, FXR agonist was reported to compete with PPAR- $\alpha$ for binding to promoters of autophagy genes and to suppress autophagy (Lee et al., 2014).

\section{AUTOPHAGY MODULATORS IN OTHER DISEASES}

Autophagy modulators have been developed for diseases other than metabolic disorders (Table 2).

\section{Neurodegenerative Diseases}

In the pathogenesis of many neurodegenerative diseases, accumulation of amyloidogenic, aggregate or misfolded proteins plays a critical role. Since such proteins are preferentially cleared by autophagy or lysosomal degradation pathway rather than proteasomal degradation pathway, potential relevance of autophagy modulators as future therapeutic agents against neurodegenerative diseases is immense (Rubinsztein, 2006). Defective autophagy or lysosomal dysfunction has been demonstrated to play a crucial pathogenic role in diverse neurodegenerative diseases such as Parkinson's disease or Alzheimer's disease (Nixon, 2007; Narendra et al., 2008; Bordi et al., 2016). In addition, aging, one of the most important risk factors for neurodegenerative diseases such as Alzheimer's disease (Thelen and Brown-Borg, 2020), diminishes autophagic activity in multiple tissues in a variety of nonmammalian and mammalian species (Sun et al., 2015; Hansen et al., 2018). For instance, the role of autophagy in aging or longevity is clearly seen in lower organisms such as C. elegans since knockdown of Beclin 1 reduced lifespan of $C$. elegans (Melendez et al., 2003). In higher or vertebrate organisms, the role of autophagy in aging is less clear. However, a couple of papers suggested an important role of autophagy in aging or longevity of mammals. Overexpression of an autophagy gene has also been reported to prolong lifespan of mice and to improve metabolic profile of aged mice (Pyo et al., 2013). Furthermore, autophagic or mitophagic activity and lysosomal function decline with aging (Hütter et al., 2007; Salminen and Kaarniranta, 2009; Sun et al., 2015; Fernando et al., 2020), while the details of the changes could be different depending on the tissue and species. These results suggest that autophagy could be intimately related to aging of mammals as well.

Given the role of autophagy deficiency or lysosomal dysfunction in the pathogenesis of neurodegenerative disorders and the close relationship between autophagy or lysosomal function and aging, autophagy enhancers could have beneficial effects against diverse neurodegenerative disorders as discussed below. Further detailed discussion regarding the role of 
TABLE 1 | Autophagy enhancers that have beneficial effects on T2D or metabolic syndrome.

\begin{tabular}{|c|c|c|c|c|}
\hline Drugs or chemicals & Mechanism and effects & $\begin{array}{l}\text { Molecular } \\
\text { targets }\end{array}$ & References & $\begin{array}{l}\text { Clinical trial } \\
\text { (phase) }\end{array}$ \\
\hline Berberine & improved metabolic profile & AMPK or SIRT1 & $\begin{array}{l}\text { Ma, et al., 2018; Sun et al., 2018; Zheng et al., } \\
2021\end{array}$ & 1,2 \\
\hline Imatinib & Improved metabolic syndrome or diabetes & $\begin{array}{l}\text { Kit or Abl; } \\
\text { Beclin } 1\end{array}$ & $\begin{array}{l}\text { Breccia et al., 2005; Han et al., 2009; Veneri } \\
\text { et al., 2005; Lim et al., } 2014\end{array}$ & - \\
\hline \multirow[t]{2}{*}{ MSL-7 } & $\begin{array}{l}\text { Enhanced clearance of lipid in the liver and } \\
\text { amelioration of metabolic inflammation }\end{array}$ & TFEB & Lim et al. (2018) & - \\
\hline & $\begin{array}{l}\text { Enhanced clearance of hIAPP oligomer and improved } \\
\beta \text {-cell function of } h I A P P^{+} \text {mice on HFD }\end{array}$ & & Kim et al. (2021b) & - \\
\hline \multirow[t]{2}{*}{ Rapamycin } & $\begin{array}{l}\text { Improved metabolic profile and } \beta \text {-cell function of Akita } \\
\text { mice }\end{array}$ & mTOR & Bachar-Wikstrom et al. (2013) & - \\
\hline & Increased longevity of mice & & Harrison et al. (2009) & 2 \\
\hline \multirow[t]{2}{*}{ Resveratrol } & $\begin{array}{l}\text { Improved metabolic profile and extended healthspan } \\
\text { of HFD-fed mice }\end{array}$ & $\mathrm{SIRT} 1$ & Baur et al. (2006) & 2 \\
\hline & Improved metabolic profile of patients with T2D & & Bo et al. (2016) & 2 \\
\hline $\operatorname{Rg} 2$ & $\begin{array}{l}\text { Improved glucose profile, insulin sensitivity and fatty } \\
\text { liver change of HFD-fed mice }\end{array}$ & - & Fan et al. (2017) & - \\
\hline \multirow[t]{3}{*}{ Spermidine } & $\begin{array}{l}\text { Improved metabolic profile and liver steatosis in HFD- } \\
\text { fed mice }\end{array}$ & EP300 elF5A & Ma et al. (2021) & - \\
\hline & $\begin{array}{l}\text { Extended lifespan of C. elegans, Drosophila and aged } \\
\text { mice }\end{array}$ & & Eisenberg et al. (2009) & - \\
\hline & $\begin{array}{l}\text { Induction of hypusination of elF5A and enhanced } \\
\text { translation of TFEB }\end{array}$ & & Zhang et al. (2019b) & - \\
\hline \multirow[t]{3}{*}{ Trehalose } & $\begin{array}{l}\text { Improved glucose profile and insulin sensitivity of } \\
\text { obese mice }\end{array}$ & TFEB; AMPK & Lim et al. (2014) & - \\
\hline & $\begin{array}{l}\text { Decreased accumulation of hIAPP oligomer and } \\
\text { amyloid in pancreatic islets }\end{array}$ & & Kim et al. (2014) & - \\
\hline & Improved fatty liver & & Zhang et al. (2020c) & - \\
\hline $\begin{array}{l}\text { Triethylene-tetramine } \\
\text { dihydrochloride (TETA) }\end{array}$ & improved metabolic profile of HFD-fed or ob/ob mice & SAT1 & Castoldi et al. (2020) & - \\
\hline Digoxin & $\begin{array}{l}\text { Beneficial metabolic effects on metabolic syndrome } \\
\text { after HFD; extension of } C \text {. elegans lifespan }\end{array}$ & TFEB & Wang et al. (2017) & 1 \\
\hline Dioscin & $\begin{array}{l}\text { Improved metabolic profile and liver steatosis of } \\
\text { obese mice }\end{array}$ & - & Liu et al. (2015a) & - \\
\hline \multirow[t]{2}{*}{ Metformin } & Protection of pancreatic $\beta$-cells against lipoapoptosis & AMPK & Jiang et al., 2014; Wu et al., 2013 & 3,4 \\
\hline & $\begin{array}{l}\text { Alleviation of aging-associated inflammation, fatty } \\
\text { liver disease or diabetic kidney disease }\end{array}$ & & $\begin{array}{l}\text { Bharath et al., 2020; Ren et al., 2019; Song } \\
\text { et al., } 2015\end{array}$ & 3 \\
\hline \multirow[t]{2}{*}{ GLP-1 agonist } & Improved liver steatosis & $\begin{array}{l}\text { AMPK or } \\
\text { cGMP }\end{array}$ & $\begin{array}{l}\text { Fang et al., 2020; Noyan-Ashraf et al., 2013; } \\
\text { Sharma et al., } 2011\end{array}$ & 4 \\
\hline & Improved diabetic nephropathy & & Yamada et al. (2021) & 4 \\
\hline SGLT2 inhibitor & Cardio- and reno-protective effects & AMPK & Packer, 2020a, Packer, 2020b & 1,4 \\
\hline \multirow[t]{2}{*}{ PPAR- $\gamma$ agonist } & Protected $\beta$-cells against lipoapoptosis & AMPK & Wu et al. (2013) & - \\
\hline & Ameliorated fatty liver disease & & Hsiao et al. (2017) & 4 \\
\hline \multirow[t]{2}{*}{ PPAR- $\alpha$ agonist } & $\begin{array}{l}\text { Alleviated HFD-induced kidney injury in a diabetic } \\
\text { kidney disease model }\end{array}$ & $\begin{array}{l}\text { Autophagy } \\
\text { genes }\end{array}$ & Sohn et al. (2017) & - \\
\hline & $\begin{array}{l}\text { Improved clinical course of clinical osteoarthritis in } \\
\text { human patients }\end{array}$ & & Nogueira-Recaldea et al. (2019) & - \\
\hline
\end{tabular}

Abbreviations: HFD, high-fat diet; TFEB, transcription factor EB. 
TABLE 2 | Autophagy enhancers that have beneficial effects on diseases other than metabolic disorders.

\begin{tabular}{|c|c|c|c|c|c|}
\hline $\begin{array}{l}\text { Drugs or } \\
\text { chemicals }\end{array}$ & $\begin{array}{c}\text { Mechanism and } \\
\text { effects }\end{array}$ & $\begin{array}{l}\text { Molecular } \\
\text { targets }\end{array}$ & Target diseases & References & $\begin{array}{c}\text { Clinical } \\
\text { trial } \\
\text { (phase) }\end{array}$ \\
\hline AUTEN-67 & Retarded disease progression & MTMR14 & $\begin{array}{l}\text { Huntington's disease or Alzheimer's } \\
\text { disease }\end{array}$ & $\begin{array}{l}\text { Billes et al., 2016; Papp } \\
\text { et al., } 2016\end{array}$ & - \\
\hline EN6 & $\begin{array}{l}\text { Accelerated clearing of TDP- } 43 \\
\text { aggregates }\end{array}$ & ATP6V1A & ALS & Chung et al. (2019) & - \\
\hline $\begin{array}{l}\text { ERRa Inverse } \\
\text { Agonist }\end{array}$ & $\begin{array}{l}\text { Enhancement of autophagosome fusion } \\
\text { to lysosome }\end{array}$ & Autolyso-some & Parkinson's disease & Suresh et al. (2018) & - \\
\hline HTT Linker & Ameliorated disease phenotype & LC3 & Huntington's disease & Li et al. (2019) & - \\
\hline ML246 & Improved memory & Beclin 2 & Alzheimer's disease & $\begin{array}{l}\text { Rocchi et al., 2017; } \\
\text { Kuramoto et al., } 2016\end{array}$ & - \\
\hline \multirow[t]{2}{*}{ ML-SA1 } & Protection of dopaminergic neurons & TFEB & ALS and Parkinson's disease & $\begin{array}{l}\text { Tedeschi et al., 2019; } \\
\text { Tsunemi et al., } 2019\end{array}$ & - \\
\hline & $\begin{array}{l}\text { Reduced apoptosis of photoreceptor } \\
\text { cells }\end{array}$ & & Retinal detachment & Yan et al. (2021) & - \\
\hline Nilotinib & $\begin{array}{l}\text { Degradation of } \alpha \text {-synuclein and reversion } \\
\text { of dopaminergic neuron loss }\end{array}$ & Beclin 1, Atg12 & Parkinson disease & Hebron et al. (2013) & 1,2 \\
\hline \multirow[t]{3}{*}{ SMER } & $\begin{array}{l}\text { Increased clearance of mutant huntingtin } \\
\text { or } \alpha \text {-synuclein }\end{array}$ & - & Huntington's disease & Sarkar et al. (2007b) & - \\
\hline & Attenuated neurodegeneration & & Alzheimer's disease & Tian et al. (2011) & - \\
\hline & Increased erythropoiesis & & Diamond-Blackfan syndrome & Doulatov et al. (2017) & - \\
\hline \multirow[t]{2}{*}{ Spermidine } & Improved cognition & PARKIN & Aging & $\begin{array}{l}\text { Lazarou et al., 2015; } \\
\text { Schroeder et al., } 2021\end{array}$ & - \\
\hline & Improved cardiac function & & Heart failure & Eisenberg et al. (2016) & - \\
\hline \multirow[t]{3}{*}{ Tat-beclin 1} & Improved cognitive function & GAPR-1 & Alzheimer's disease & Shoji-Kawata et al. (2013) & - \\
\hline & $\begin{array}{l}\text { Beneficial effects on cardiac function } \\
\text { after TAC }\end{array}$ & & Heart failure & Shirakabe et al. (2016) & - \\
\hline & $\begin{array}{l}\text { Reduced microbial replication and } \\
\text { improved clinical outcome of infection }\end{array}$ & & $\begin{array}{l}\text { Listeria monocytogenes; Sindvis virus, } \\
\text { chikungunya virus, West Nile virus, HIV }\end{array}$ & Shoji-Kawata et al. (2013) & - \\
\hline \multirow[t]{3}{*}{ Trehalose } & $\begin{array}{l}\text { Reduced } A \beta \text { deposition and improved } \\
\text { cognitive deficit and learning disability }\end{array}$ & TFEB & Alzheimer's disease & Du et al. (2013) & 1 \\
\hline & $\begin{array}{l}\text { Reduced atherosclerotic plaque burden } \\
\text { in ApoE-KO mice fed western diet }\end{array}$ & & Atherosclerosis & Sergin et al. (2017) & 2 \\
\hline & Attenuated inflammation of the brain & & Mucopolysacchari-dosis IIIb & Lotfi et al. (2018) & - \\
\hline UMI-77 & Ameliorated neurological deficit & MCL-1 & Alzheimer's disease & Cen et al. (2020) & - \\
\hline Felodipine & Neuroprotection & - & Huntington's disease & Siddiqi et al. (2019) & - \\
\hline Rilmenidine & Amelioration of neurological signs & - & Huntington's disease & Rose et al. (2010) & - \\
\hline Lithium & $\begin{array}{l}\text { Clearance of } \alpha \text {-synuclein aggregate and } \\
\text { mutant Huntingtin }\end{array}$ & $\begin{array}{l}\text { Inositol } \\
\text { monophos- } \\
\text { phatase }\end{array}$ & $\begin{array}{l}\text { Parkinson's disease; Huntington's } \\
\text { disease }\end{array}$ & Sarkar et al. (2005) & 1,2 \\
\hline \multirow[t]{2}{*}{ GLP-1 agonists } & Improved motor function & mTOR & Parkinson disease & Zhang et al. (2020a) & - \\
\hline & Attenuated fibrosis after aortic banding & & Heart Diseases & Zheng et al. (2019) & - \\
\hline PPAR- $\alpha$ agonist & Improved cognition & Autophagy genes & Alzheimer's disease & Luo et al. (2020) & 1 \\
\hline Sildenafil & Improved hear failure & PGK1 & Heart Diseases & Ranek et al. (2019) & 4 \\
\hline \multirow[t]{2}{*}{ Metformin } & Ameliorated ultrastructural abnormalities & AMPK & Diabetic Heart Diseases & Xie et al. (2011) & \\
\hline & Autophagy induction & & Diabetic kidney disease & Kaushal et al. (2020) & 4 \\
\hline Fenofibrate & $\begin{array}{l}\text { Prevention of fibrosis in an animal model } \\
\text { of T1D }\end{array}$ & Autophagy genes & Heart Diseases & Zhang et al. (2016) & - \\
\hline
\end{tabular}


TABLE 2 | (Continued) Autophagy enhancers that have beneficial effects on diseases other than metabolic disorders.

\begin{tabular}{|c|c|c|c|c|c|}
\hline $\begin{array}{l}\text { Drugs or } \\
\text { chemicals }\end{array}$ & $\begin{array}{l}\text { Mechanism and } \\
\text { effects }\end{array}$ & $\begin{array}{l}\text { Molecular } \\
\text { targets }\end{array}$ & Target diseases & References & $\begin{array}{c}\text { Clinical } \\
\text { trial } \\
\text { (phase) }\end{array}$ \\
\hline Artemisinin & Alleviation of atherosclerosis & AMPK & Atherosclerosis & Cao et al. (2019) & - \\
\hline Fucoidan & Alleviation of atherosclerosis & TFEB & Atherosclerosis & Cheng et al. (2020) & - \\
\hline Rapamycin & $\begin{array}{l}\text { Ameliorated damage after coronary } \\
\text { ligation }\end{array}$ & mTOR & Ischemic heart disease & Sciarretta et al. (2012) & - \\
\hline Rapamycin & $\begin{array}{l}\text { Suppressed photoreceptor degeneration } \\
\text { or inflammation }\end{array}$ & mTOR & Retinitis & Okamoto et al. (2016) & - \\
\hline Temsirolimus & $\begin{array}{l}\text { Protected against sepsis-induced acute } \\
\text { renal failure }\end{array}$ & mTOR & Kidney Diseases & Howell et al. (2013) & 2 \\
\hline Urolithin A & Ameliorated I/R-induced injury & TFEB & Kidney Diseases & Wang et al. (2019b) & \\
\hline Atrasentan & $\begin{array}{l}\text { Amelioration of diabetic kidney disease } \\
\text { and enhancement of Foxo1 expression }\end{array}$ & miR-21 & Kidney Diseases & Wang et al. (2019a) & 2 \\
\hline Klotho & Ameliorated FK506-induced injury & TFEB & Kidney Diseases & Lim et al. (2019) & - \\
\hline A77 1726 & Restriction of bacterial growth & AMPK & Infection (Salmonella) & Zhuang et al. (2020) & - \\
\hline Flubendazole & $\begin{array}{l}\text { Promoted clearance of intracellular } \\
\text { bacteria }\end{array}$ & mTOR & Infection (HIV) & Chauhan et al. (2015) & - \\
\hline BC18630 & Reduced viral load and lung injury & TFEB & Infection (SARS-CoV-2) & Liu et al. (2021) & - \\
\hline $\begin{array}{l}\text { 2-hydroxypropy } 1-\beta \text { - } \\
\text { cyclodextrin }\end{array}$ & $\begin{array}{l}\text { Accelerated clearance of proteolipid } \\
\text { aggregates }\end{array}$ & TFEB & Neuronal ceroid lipofuscinosis & Song et al. (2014) & - \\
\hline Rosiglitazone & Attenuated post-operative fibrosis & Beclin 1 & Glaucoma & Zhang et al. (2019a) & - \\
\hline Artesunate (ART) & Ameliorated retinopathy & AMPK/SIRT1 & Diabetic retinopathy & Li et al. (2021) & - \\
\hline Apigenin & $\begin{array}{l}\text { Improved clinical index and reduce } \\
\text { intraocular oxidative damage }\end{array}$ & Nrf2 & Age-related macular degeneration & Zhang et al. (2020b) & - \\
\hline Latrepirdine & Improved cognition & mTOR & Alzheimer's disease & Steele and Gandy, (2013) & - \\
\hline Calpastatin & $\begin{array}{l}\text { Improved motor function and decrease } \\
\text { tremor }\end{array}$ & Calpain & Huntington's disease & Menzies et al. (2015) & - \\
\hline 6-Bio & $\begin{array}{l}\text { Improved motor coordination and } \\
\text { locomotion }\end{array}$ & GSK3b & Parkinson's disease & Suresh et al. (2017) & - \\
\hline Vitamin D & Inhibited viral replication & Beclin 1 & HIV infection & $\begin{array}{l}\text { Campbell and Spector, } \\
\text { (2011) }\end{array}$ & 2,3 \\
\hline Acacetin & Reduced bacterial burden in vivo & TFEB & Salmonella & $\begin{array}{l}\text { Ammanathan et al. } \\
(2020)\end{array}$ & - \\
\hline
\end{tabular}

Abbreviations: ALS, amyotrophic lateral sclerosis; T1D, Type 1 diabetes; TAC, Transverse aortic constriction.

autophagy specifically in neurodegenerative diseases can be found in recent review articles (Rahman and Rhim, 2017; Park et al., 2020). Several autophagy enhancers against neurodegenerative diseases not covered in the text can be found in Table 2 .

\section{AUTEN-67}

AUTEN-67 (Autophagy Enhancer-67), an MTMR14 inhibitor, has been reported to impede progression of the diseases in a Drosophila model of Huntington's disease and in a mouse model of Alzheimer's disease (Billes et al., 2016; Papp et al., 2016).

\section{EN6}

A small molecule autophagy activator (EN6) that covalently targets cysteine 277 of ATP6V1A subunit of lysosomal v-ATPase was identified in a screening using cysteine- and lysine-reactive ligands. EN6 decoupled v-ATPase from the Ragulator-Rag complex and induced mTORC1 inhibition, leading to accelerated clearing of TDP-43 aggregates through autophagy enhancement (Chung et al., 2019).

\section{ERR a Inverse Agonist}

It was reported that overexpression of ERRa inhibits autophagosome fusion to lysosome (Suresh et al., 2018). XCT 790, an inverse agonist of ERRa, was shown to exert neuroprotective effects in a preclinical model of Parkinson's disease through autophagy upregulation (Suresh et al., 2018).

\section{HTT Linker}

Recently, compounds linking target substrates to LC3 have been developed. An HTT-LC3 linker has been reported to ameliorate 
Huntington's disease phenotype by tethering mutant Huntingtin to LC3 ( $\mathrm{Li}$ et al., 2019). This compound belongs to the class of autophagosome tethering compounds (ATTECs).

\section{ML246}

ML246, a brain-permeable autophagy enhancer, has been shown to enhance clearance of $A \beta$, protect neuronal cells from $A \beta$ induced cell death, and improve memory of Alzheimer's disease mice, which was associated with a trend toward reduced brain amyloid accumulation (Rocchi et al., 2017). ML246 has also been shown to prevent cannabinoid tolerance by inhibiting Beclin 2 (BECN2) binding to GRASP1, a receptor for cannabinoid receptor 1 (CNR1) degradation (Kuramoto et al., 2016), which might be employed to enhance medical effects of cannabinoid such as analgesia.

\section{ML-SA1}

Transient receptor potential cation channel, mucolipin subfamily 1 (TRPML1) channel is a lysosomal $\mathrm{Ca}^{2+}$ exit channel that is important in activation of TFEB, a master regulator of autophagy gene expression and lysosomal biogenesis (Wang et al., 2015). ML-SA1, an agonist of TRPML channel (Shen et al., 2012), has been reported to protect dopaminergic neurons in cell models of amyotrophic lateral sclerosis (ALS) and Parkinson's disease by boosting autophagy or enhancing lysosomal $\mathrm{Ca}^{2+}$ exocytosis (Tedeschi et al., 2019; Tsunemi et al., 2019).

\section{Nilotinib}

As an Abl inhibitor related to aforementioned Imatinib, Nilotinib has been shown to induce degradation of a-synuclein and reverse loss of dopaminergic neurons through autophagy activation which was associated with increased levels of Beclin 1 and Atg12 (Hebron et al., 2013). Radotinib, a related compound, has also been reported to be neuroprotective in a preclinical Parkinson disease model (Lee et al., 2018). However, recent phase 2 clinical trials using Nilotinib showed mostly negative results (Simuni et al., 2021). A recent paper also reported no effect of Nilotinib on R6/2, an animal model of Huntington's disease (Kumar et al., 2021).

\section{SMER}

SMERs were initially selected by small-molecule screening in yeast and, their activity on mammalian autophagy was confirmed employing a cell system overexpressing A53T asynuclein, an autophagy substrate. SMER10 (aminopyrimidone), SMER18 (vinylogous amide), SMER28 (bromo-substituted quinazoline) and their analogs increased autophagic clearance of mutant huntingtin or a-synuclein in vitro in an-mTOR-independent manner, and also attenuated neurodegeneration in D. melanogaster model of Huntington's disease (Sarkar et al., 2007b). Later studies showed that SMER28 can accelerate in vitro clearance of $A \beta$ peptide or carboxy terminal fragment of amyloid precursor protein (APP-CTF) (Tian et al., 2011). Besides neurodegenerative disorders, SMER28 has been reported to increase erythropoiesis in cell or animal models of Diamond-
Blackfan syndrome, a disorder of abnormal erythroid progenitor differentiation, through Atg5 (Doulatov et al., 2017).

\section{Spermidine}

Aforementioned spermidine was recently reported to improve cognitive dysfunction in aged mice, which was associated with increased mitochondrial respiration and dependent on Atg7 and PINK1, a kinase inducing mitophagy through recruitment of PARKIN onto dysfunctional mitochondria (Lazarou et al., 2015; Schroeder et al., 2021).

\section{Tat-Beclin 1}

HIV Nef-interacting domain of Beclin 1 attached to Tat transduction domain (Tat-beclin 1) could enhance autophagic flux by binding to GAPR-1, an autophagy inhibitor, and liberating Beclin 1 from Golgi complex (Shoji-Kawata et al., 2013) (Figure 1). Tat-beclin 1 has been reported to accelerate clearance of htt103Q aggregate or $A \beta$ oligomer and to improve cognitive function of Alzheimer's disease model mice (Shoji-Kawata et al., 2013). In contrast, a high dose of Tat-beclin 1 has been reported to induce autosis, a special form of cell death characterized by high autophagic activity, inhibition by $\mathrm{Na}^{+} / \mathrm{K}^{+}$ ATPase inhibitors and unique morphological features including focal rupture of plasma membrane, electron-dense swollen mitochondria and ballooning of perinuclear space (Liu et al., 2013). Furthermore, Tat-Beclin 1 peptide has been shown to aggravate hypoxic injury of the rat brain, which might be related to the inhibition of autophagosome-lysosome fusion due to elevated expression of Rubicon (Nah et al., 2020).

\section{Trehalose}

Trehalose, a disaccharide with autophagy enhancing activity as discussed above, was also shown to exert effects on neurodegeneration and aging. Trehalose could prolong total and reproductive life span, retard decrease in pharyngeal pumping due to aging, enhance thermotolerance and suppress polyQ aggregation in C. elegans, while the role of autophagy in trehalose effects was not directly demonstrated in this paper (Honda et al., 2010). Trehalose also reduced $A \beta$ deposition and improved cognitive deficit and learning disability in a mouse model of Alzheimer's disease (Du et al., 2013).

\section{UMI-77}

In a screening using an FDA-approved drug library, UMI-77, a BH3mimetic for MCL-1, was identified as an inducer of mitophagy without effects on apoptosis. MCL-1 was suggested to be a mitophagy receptor, and UMI-77 ameliorated neurological deficit in a mouse model of Alzheimer's disease (Cen et al., 2020).

\section{Other Previously Known Chemicals or Drugs}

In several screening for autophagy modulators, some known chemicals or drugs have been identified and shown to enhance clearance of aggregate or amyloid proteins associated with neurodegeneration. For instance, GFP-LC3-based screening followed by assay of FYVE-RFP ${ }^{+}$vesicle identified eight known chemicals inducing autophagic degradation of long-lived proteins in an mTOR-independent manner (Fluspirilene, trifluoperazine, 
pimozide, nicardipine, penitrem A, niguldipine, loperamide and amiodarone). Those chemicals also reduced accumulation of polyQ (Zhang et al., 2007).

Another autophagy enhancer screening using EGFP-LC3 vesicles identified verapamil, valproate and clonidine as autophagy enhancers acting through decreased $\left(\mathrm{Ca}^{2+}\right)_{\mathrm{i}}$ or intracellular inositol 1,4,5-trisphosphate $\left(\mathrm{IP}_{3}\right)$ content and calpain inhibition (Williams et al., 2008). Such chemicals have been reported to delay Huntington's disease-like phenotypes in Drosophila or zebrafish models. A similar screening of autophagy enhancer using GFP-RFP-LC3 system and a chemical library identified flubendazole, an anti-helminthic agent, and bromhexine as autophagy enhancers. Flubendazole led to disrupted dynamic microtubule, followed by displacement and inhibition of mTOR. Flubendazole and bromhexine were also reported to reduce accumulation of total and hyperphosphorylated Tau (Chauhan et al., 2015). Felodipine, an anti-hypertensive drug acting on L-type $\mathrm{Ca}^{2+}$ channel similar to verapamil, has also been reported to have autophagy-enhancing and neuroprotective effects in animal models of Huntington's disease (Siddiqi et al., 2019). Rilmenidine, another anti-hypertensive drug, has been reported to enhance autophagy and to ameliorate neurological signs in an animal model of Huntington's disease (Rose et al., 2010).

Lithium, a well-known drug for bipolar disease, has been reported to induce autophagy through inhibition of inositol monophosphatase and depletion of free inositol or $\mathrm{IP}_{3}$. Lithium has been shown to induce clearance of a-synuclein aggregate or mutant Huntingtin (Sarkar et al., 2005). A recent paper showed the effect of lithium in the clearance of Tau aggregate (Uddin et al., 2021).

GLP-1 agonists, aforementioned anti-diabetic drugs with potential autophagy enhancing activity, have also been reported to confer therapeutic effects on Parkinson disease animal models (Zhang L. et al., 2020).

PPAR- $\alpha$ agonist such as gemfibrozil or Wy14643 has been reported to ameliorate Alzheimer's disease-related phenotype in a murine model through autophagy induction (Luo et al., 2020).

\section{Heart Diseases}

Autophagy is crucial in the development of the heart and in the maintenance of cardiac function (Gatica et al., 2021). Dysregulated autophagy has been reported to be involved in the pathogenesis of several cardiovascular diseases.

As discussed above, spermidine induced autophagy through epigenetic regulation of autophagy genes (Pietrocola et al., 2015), and could extend lifespan of experimental animals (Eisenberg et al., 2016). Spermidine has also been reported to improve cardiac function in age- or hypertension-associated heart failure models (Eisenberg et al., 2016).

Sildenafil, an inhibitor of phosphodiesterase type-5, can activate protein kinase G1 (PGK1) that has been shown to phosphorylate TSC2 and induce autophagy through mTORC1 inhibition (Ranek et al., 2019). Sildenafil has been reported to reverse autophagy inhibition and heart failure after cardiac pressure overload in a phosphorylation-silencing Tsc2 mutantknockin mouse model (Ranek et al., 2019).

Everolimus, a relatively selective inhibitor of mTORC1, also had similar effects on failing heart in the same mice.

Metformin, a well-known anti-diabetic drug, has been reported to enhance cardiac autophagic activity and ameliorates cardiac ultrastructural abnormalities associated with diabetes through AMPK activation in an animal model of diabetic cardiomyopathy (Xie et al., 2011).

GLP-1 agonists have also been reported to have therapeutic effects on myocardial fibrosis after aortic banding through autophagy enhancement (Zheng et al., 2019).

Fenofibrate, a classical PPAR- $\alpha$ agonist, has been reported to prevent cardiac fibrosis in an animal model of type 1 diabetes (T1D), accompanied by enhanced autophagic activity in vivo (Zhang et al., 2016).

Tat-Beclin 1, aforementioned autophagy enhancer liberating beclin 1 from Golgi complex, was shown to confer beneficial effects on heart failure after transverse aortic constriction (TAC) (Shirakabe et al., 2016), which is consistent with a previous report that cardiac autophagy plays a protective role in ischemic cardiac disease of mice with diet-induced obesity (Sciarretta et al., 2012). On the other hand, Tat-Beclin 1 peptide has been shown to aggravate cardiac ischemia-reperfusion (I/R)-induced cardiac injury and autosis, which was accompanied by increased expression of Rubicon inhibiting multiple steps of autophagy including autophagosome-lysosome fusion (Nah et al., 2020).

Another example showing deleterious effect of autophagy on cardiac tissue has been published. A paper reported that dimethyl a-ketoglutarate inhibited mal-adaptive cardiac autophagy and suppressed TAC-induced heart failure in a pressure-overloadinduced cardiomyopathy model after conversion to cytosolic acetyl CoA acting on EP300, an acetyltransferase (Mariño et al., 2014).

In addition to heart failure, atherosclerosis is another heart disease that is closely related to autophagy, particularly that of macrophages (Liao et al., 2012). Trehalose, an aforementioned autophagy enhancer, could reduce atherosclerotic plaque burden in $A p o E-\mathrm{KO}$ mice fed western diet by enhancing autophagy (Sergin et al., 2017).

Spermidine, an autophagy enhancer discussed above, has also been reported to attenuate atherosclerosis and to inhibit necrotic core formation through autophagy activation (Michiels et al., 2016).

Artemisinin is an endoperoxide sesquiterpene lactone with multiple beneficial effects such as anti-malarial, antiinflammation and anti-oxidant effects. Artemisinin has also been reported to attenuate atherosclerosis in HFD-fed ApoE$\mathrm{KO}$ mice by enhancing autophagic activity of macrophages (Cao et al., 2019).

Fucoidan, a marine sulfated polysaccharide derived from brown seaweeds with anti-inflammatory activity, has also been reported to alleviate atherosclerosis in HFD-fed ApoE-KO mice by enhancing autophagic activity (Cheng et al., 2020).

In ischemic heart disease, Rapamycin, a classical mTOR inhibitor and autophagy enhancer, could ameliorate cardiac damage after coronary ligation in mice fed HFD by enhancing 
autophagy and protecting against cardiomyocyte death (Sciarretta et al., 2012).

Further detailed discussion regarding the role of autophagy specifically in cardiovascular diseases can be found in recent review articles (Lampert and Gustafsson, 2018; Gatica et al., 2021)

\section{Kidney Diseases}

Kidney is a critical target organ of diabetic complication. Previous papers showed the role of altered autophagy in the development of several kidney diseases including diabetic nephropathy and kidney fibrosis (Liu W. J. et al., 2015; Nam et al., 2019; Zhao et al., 2019). Several autophagy enhancers have been administered to such diverse kidney disease models.

Temsirolimus, an autophagy enhancer belonging to mTORC1 inhibitor family, has been administered to septic kidney disease model with beneficial clinical effects accompanied by autophagy enhancement (Howell et al., 2013). A variety of authentic or potential AMPK activators such as metformin, $\omega-3$ polyunsaturated fatty acids, quercetin, neferine, astragaloside IV, mangiferin, cinacalcet, berberine, progranulin have also been administered to diabetic kidney disease, cisplatin-induced nephropathy or I/R kidney injury, leading to clinical improvement and autophagy enhancement (Kaushal et al., 2020).

Aforementioned autophagy enhancers such as fenofibrate, pioglitazone and SGLT2 inhibitors have also been employed to treat diabetic kidney disease or I/R kidney injury with clinical improvement.

Urolithin A, the main metabolite in pomegranate juice, has been reported to ameliorate I/R-induced kidney injury through TFEB activation (Wang Y. et al., 2019).

Atrasentan, an antagonist of endothelin 1 receptor subtype A $\left(\mathrm{ET}_{\mathrm{A}}\right)$, was able to ameliorate diabetic kidney disease, by downregulating miR-21 expression, enhancing Foxo1 expression and upregulating autophagy (Wang J. et al., 2019).

Klotho, an obligate co-receptor for fibroblast growth factor 23 (FGF23), has also been reported to ameliorate FK506-induced renal injury with impaired lysosomal function by inducing TFEB activation through inhibition of GSK3 $\beta$ phosphorylation (Lim et al., 2019).

Further detailed discussion regarding the role of autophagy specifically in the kidney diseases can be found in recent review articles (Tang et al., 2020; Zheng et al., 2020).

\section{Infection}

Autophagy is also important antimicrobial defense mechanism against diverse infectious agents such as Mycobacterium tuberculosis, Salmonella or Streptococcus. Autophagy can contain cytosolic bacteria escaping from endosome/vacuole and induce maturation of phagosome into phagolysosome (Gutierrez et al., 2004; Nakagawa et al., 2004; Wild et al., 2008). In the intestine, secretory autophagy from Paneth cells mediates release of lysozyme, a critical antimicrobial peptide, and is an important host response against bacterial infection, which is defective in patients with Crohn's disease (Bel et al., 2017).

In a paper studying the effect of autophagy enhancer on infection, Tat-beclin 1 reduced replication of Listeria monocytogenes and viruses such as Sindbis virus, chikungunya virus, West Nile virus and HIV in vitro and improved clinical outcome of such infections in vivo (Shoji-Kawata et al., 2013).

A77 1726, an active metabolite of anti-inflammatory drug leflunomide, has been reported to restrict Salmonella growth through AMPK activation and autophagy enhancement (Zhuang et al., 2020).

SMER 28 has also been reported to have capability to kill Mycobacterium (Floto et al., 2008).

Flubendazole, an autophagy enhancer identified in a screening using GFP-RFP-LC3 system and a chemical library, inhibited HIV transfer from dendritic cells to $\mathrm{T}$ cells and promoted clearance of intracellular bacteria (Chauhan et al., 2015).

Everolimus or rapamycin has also been reported to suppress productive infection of HIV (Cloherty et al., 2021).

Intriguingly, therapeutic effect of a novel TFEB activator against COVID-19 was reported in a preprint paper. In corona virus infection, TFEB was shown to be degraded through proteasomal degradation. BC18630 selected by an in silico screening of inhibitors against DCAF7, a putative E3 ligase mediating degradation of TFEB, was shown to reduce viral load and lung injury in a Syrian hamster model of SARS-CoV-2 infection (Liu et al., 2021). Modulators of autophagy, lysosome or TFEB could have therapeutic effects against COVID-19 pandemic caused by infection with SARS-CoV-2 that egresses host cells through lysosomal trafficking and disrupts lysosomal function (Ghosh et al., 2020).

In contrast to the protective role of autophagy against infectious diseases, some pathogens such as $H$. pylori or uropathogenic $E$. coli have been reported to hijack host autophagic machinery for their survival and growth (Huab et al., 2020). In this case, inhibition of autophagy may have a therapeutic effect.

\section{Lysosomal Storage Diseases}

LSDs are a group of rare diseases in which lysosomal function is primarily impaired due to genetic causes and characterized by accumulation of excessive substrates in lysosome, leading to lysosomal dysfunction and a variety of systemic manifestations including neurodegeneration (Darios and Stevanin, 2020). As lysosomal dysfunction and autophagy impairment are observed in most of the LSDs, enhancement of autophagic or lysosomal activity could be a new modality to treat such diseases.

2-hydroxypropyl- $\beta$-cyclodextrin, an excipient and cholesterol-extracting agent, has been shown to activate TFEB and accelerate clearance of proteolipid aggregates in cells from patients with neuronal ceroid lipofuscinosis, a LSD (Song et al., 2014).

Trehalose has also been reported to attenuate inflammation of the brain and retina and to improve vision by activating TFEB and autophagic activity in a mouse model of mucopolysaccharidosis IIIB (MPS IIIB), a LSD caused by mutation of $\alpha-N$-acetylglucosaminidase (NAGLU) (Lotfi et al., 2018).

\section{Ocular Diseases}

The risk of eye diseases such as macular degeneration is exponentially increasing in advanced age (Luu and Palczewski, 
2018), which might be related to dysregulated autophagy in aging (Kivinen, 2018).

Rosiglitazone has also been reported to attenuate postoperative fibrosis in an animal model of glaucoma, which was associated with autophagy enhancement (Zhang F. et al., 2019).

Artesunate (ART) is a semi-synthetic derivative of aforementioned artemisinin, has also been reported to ameliorate diabetic retinopathy through AMPK/SIRT1 activation (Li et al., 2021).

ML-SA1, aforementioned agonist of lysosomal TRPML1 Ca ${ }^{2+}$ channel, has been shown to protect retinal structure, reduce apoptosis of photoreceptor cells and improve vision-dependent behavior in a rat model of retinal detachment, which was accompanied by enhanced autophagy (Yan et al., 2021).

Apigenin, a well-known antioxidant and anti-inflammatory flavonoid, has been reported to improve clinical index and reduce intraocular oxidative damage in a mouse model of age-related macular degeneration (AMD) by upregulating autophagy and expression of $\mathrm{Nrf2}$, a master regulator of anti-oxidative gene expression (Zhang et al., 2020c).

Effects of Rapamycin in an animal model of endotoxininduced uveitis and retinitis model have also been studied, which demonstrated suppression photoreceptor degeneration and inflammation together with inhibition of NF- $\mathrm{BB}$ or mTOR and enhancement of autophagy (Okamoto et al., 2016).

\section{AUTOPHAGY INHIBITOR}

Inhibitors of autophagy have been developed mostly for application in cancer. Autophagy has been reported to inhibit or accelerate the development of cancer, depending on the stage of carcinogenesis as summarized in the review (White, 2012). Thus, autophagy deficiency could promote the initiation of cancer by producing reactive oxygen species and inducing chromosomal abnormality. In a similar vein, cell death due to autophagy hyperactivation or autophagic cell death has been reported to limit chromosomal instability during replicative crisis causing telomeric DNA damage (Nassour et al., 2019). Some autophagy genes act as tumor suppressor genes (Qu et al., 2003; Liang et al., 2006; Cianfanelli et al., 2015). At the later stage of cancer development, autophagy can accelerate the progression of cancer by supplying amino acids or energy that is necessary for the progression of cancer through tumor cell-autonomous or non-tumor cell-autonomous manner (Kimmel and White, 2017). Autophagy of host cells might play a role in the maintenance of serum arginine level that is necessary for tumor growth (Poillet-Perez et al., 2021).

Several autophagy inhibitors have been developed as potential anti-cancer drugs such as chloroquine or ULK1 inhibitors (Chude and Amaravadi, 2017; Martin et al., 2018), exploiting metabolic requirement of cancer cells. For instance, chloroquine in conjunction with leucine-free diet has been reported to suppress growth of melanoma that is resistant to mTORC1 inhibition and autophagy activation by leucine deprivation (Sheen et al., 2011). In contrast, the effect of ULK1/2 inhibition has been questioned since abrogation of ULK1/2mediated autophagy could not block extracellular protein- dependent cancer cell growth (Palm et al., 2015). Further discussion regarding the role of autophagy in the pathogenesis of cancer or that of autophagy modulators in the cancer treatment is beyond the scope of this article, and the readers are encouraged to consult excellent review papers (White, 2012; Amaravadi et al., 2017; Levy et al., 2017; Miller and Thorburn, 2021).

\section{NEW APPROACHES FOR AUTOPHAGIC OR LYSOSOMAL DEGRADATION OF SELECTIVE TARGETS}

Recently, novel autophagic or lysosomal protein degradation techniques such as lysosome targeting chimera (LYTAC), autophagy-targeting chimera (AUTAC) or autophagy-tethering compound (ATTEC) have been developed (Ding et al., 2021). LYTAC comprises an antibody fused to mannose-6-phosphate (M6P) that can be recognized by cation-independent M6P receptor (CI-M6PR), a lysosomal trafficking receptor (Banik et al., 2020). Using this method, extracellular targets such as apolipoprotein E (APOE) or plasma membrane-bound targets such as epidermal growth factor receptor (EGFR) could be targeted to endosome or lysosome for degradation. AUTAC consists of warheads to selective targets, linker and guanine degradation tag inducing S-guanylation-mediated autophagic degradation (Takahashi et al., 2019). This technique may allow degradation of large molecules, protein aggregates, organelles such as mitochondria or bacteria which has not been possible with PROTAC (proteolysis-targeting chimera) utilizing ubiquitin E3 ligase-binding ligands (Ding et al., 2021). ATTEC employs compounds interacting with both mutant huntingtin (HTT) protein targets and LC3, directing target proteins to autophagic degradation without ubiquitination ( $\mathrm{Li}$ et al., 2019). These novel approaches will provide outstanding tools for the development of next-generation modulators of autophagy or lysosomal degradation.

\section{CONCLUSION AND FUTURE PERSPECTIVES}

While the significance of autophagy in the physiological function and pathogenesis of diverse diseases or aging is becoming more and more clear, authentic pharmacological autophagy modulators are still not available in the clinics except drugs that have already been used but were found to have autophagy-modulating effects later. Pharmacological modulation of specific processes or molecules among a wide array of the related genes or proteins at the specific sites is still not possible, precluding the use of autophagy modulators in the current circumstances. Lack of reliable biomarkers and suitable assay systems for measurement of autophagic flux in humans is also a big hurdle. Nonetheless, as detailed molecular mechanisms of selective autophagy are being discovered, specific modulation of specialized aspects of autophagy possibly at the specific tissues or sites will not be a remote possibility, which will be of a paramount importance for clinical application of autophagy modulators.

However, some caution might be exercised in the use of autophagy enhancer for treatment of human diseases. 
Lysosomal dysfunction is frequently observed in diverse tissues of patients with Alzheimer's disease, obesity, T2D and associated conditions or aging (Hütter et al., 2007; Liu W. J. et al., 2015; Bordi et al., 2016; Zhao et al., 2019; Fernando et al., 2020; Kim et al., 2021a). In such conditions, accumulation of autophagic intermediates due to lysosomal dysfunction may be further increased after administration of autophagy enhancers activating earlier steps of autophagy, which might lead to autophagic stress or autophagic cell death (Zheng et al., 2020). Thus, it might be necessary to manage lysosomal dysfunction together with or before administration of autophagy enhancers. Possibility of aggravation of pre-existing cancer or infection due to pathogens exploiting host autophagic machinery for their survival by autophagy enhancers should also be kept in mind. Therefore, maintaining the exquisite balance of timing, duration and the extent of autophagy modulation depending on the types and stages of the diseases is crucial.

\section{REFERENCES}

Agudo-Canalejo, J., Schultz, S. W., Chino, H., Migliano, S. M., Saito, C., and Koyama-Honda, I. (2021). Wetting Regulates Autophagy of Phase-Separated Compartments and the Cytosol. Nature 591, 142-146. doi:10.1038/s41586-0202992-3

Alsaleh, G., Panse, I., Swadling, L., Zhang, H., Richter, F. C., Meyer, A., et al. (2020). Autophagy in T Cells from Aged Donors Is Maintained by Spermidine and Correlates with Function and Vaccine Responses. eLife 9, e57950. doi:10.7554/ eLife.57950

Amaravadi, R. K., Kimmelman, A. C., and Debnath, J. (2017). Targeting Autophagy in Cancer: Recent Advances and Future Directions. Cancer Discov. 9, 1167-1181.

Ammanathan, V., Mishra, P., Chavalmane, A. K., Muthusamy, S., Jadhav, V., Siddamadappa, C., et al. (2020). Restriction of Intracellular Salmonella Replication by Restoring TFEB-Mediated Xenophagy. Autophagy 16, 1684-1697. doi:10.1080/15548627.2019.1689770

Ardestani, A., Lupse, B., Kido, Y., Leibowitz, G., and Maedler, K. (2018). mTORC1 Signaling: A Double-Edged Sword in Diabetic $\beta$ Cells. Cell Metab. 27, 314-331. doi:10.1016/j.cmet.2017.11.004

Bachar-Wikstrom, E., Wikstrom, J. D., Ariav, Y., Tirosh, B., Kaiser, N., Cerasi, E., et al. (2013). Stimulation of Autophagy Improves Endoplasmic Reticulum Stress-Induced Diabetes. Diabetes 62, 1227-1237. doi:10.2337/db12-1474

Banik, S. M., Pedram, K., Wisnovsky, S., Ahn, G., Riley, N. M., and Bertozzi, C. R. (2020). Lysosome-targeting Chimaeras for Degradation of Extracellular Proteins. Nature 584, 291-297. doi:10.1038/s41586-020-2545-9

Barlow, A. D., Nicholson, M. L., and Herbert, T. P. (2013). Evidence for Rapamycin Toxicity in Pancreatic B-Cells and a Review of the Underlying Molecular Mechanisms. Diabetes 62, 2674-2682. doi:10.2337/db13-0106

Baur, J. A., Pearson, K. J., Price, N. L., Jamieson, H. A., Lerin, C., Kalra, A., et al. (2006). Resveratrol Improves Health and Survival of Mice on a High-Calorie Diet. Nature 444, 337-342. doi:10.1038/nature05354

Bel, S., Pendse, M., Wang, Y., Li, Y., Ruhn, K. A., Hassell, B., et al. (2017). Paneth Cells Secrete Lysozyme via Secretory Autophagy during Bacterial Infection of the Intestine. Science 357, 1047-1052. doi:10.1126/science.aal4677

Bellodi, C., Lidonnici, M. R., Hamilton, A., Helgason, G. V., Soliera, A. R., Ronchetti, M., et al. (2009). Targeting Autophagy Potentiates Tyrosine Kinase Inhibitor-Induced Cell Death in Philadelphia Chromosome-Positive Cells, Including Primary CML Stem Cells. J. Clin. Invest. 119, 1109-1123. doi:10.1172/jci35660

Bharath, L. P., Agrawal, M., McCambridge, G., Nicholas, D. A., Hasturk, H., Liu, J., et al. (2020). Metformin Enhances Autophagy and Normalizes Mitochondrial Function to Alleviatea Aging-Associated Inflammation. Cel. Metab. 32, 44-55. doi:10.1016/j.cmet.2020.04.015

\section{AUTHOR CONTRIBUTIONS}

MS-L conceived the paper. KP and MS-L wrote the manuscript. All authors approved the submitted version.

\section{FUNDING}

This study was supported by a National Research Foundation of Korea (NRF) grant funded by the Korea government (MSIT) (NRF-2019R1A2C3002924) and by the Bio\&Medical Technology Development Program (2017M3A9G7073521). M-SL is the recipient of a grant from the Faculty Research Assistance Program of Yonsei University College of Medicine (6-20160055) and A3 Foresight Program of the NRF (2015K2A2A6002060).

Billes, V., Kovács, T., Hotzi, B., Manzéger, A., Tagscherer, K., Komlós, M., et al. (2016). AUTEN-67 (Autophagy Enhancer-67) Hampers the Progression of Neurodegenerative Symptoms in a Drosophila Model of Huntington's Disease. J. Huntingtons Dis. 5, 133-147. doi:10.3233/jhd-150180

Bo, S., Gambino, R., Ponzo, V., Cioffi, I., Goitre, I., Evangelista, A., et al. (2018). Effects of Resveratrol on Bone Health in Type 2 Diabetic Patients. A DoubleBlind Randomized-Controlled Trial. Nutr. Diabetes 6, 51. doi:10.1038/s41387018-0059-4

Bo, S., Ponzo, V., Ciccone, G., Evangelista, A., Saba, F., Goitre, I., et al. (2016). Six Months of Resveratrol Supplementation Has No Measurable Effect in Type 2 Diabetic Patients. A Randomized, Double Blind, Placebo-Controlled Trial. Physiol. Res. 111, 896-905. doi:10.1016/j.phrs.2016.08.010

Bordi, M., Berg, M. J., Mohan, P. S., Peterhoff, C. M., Alldred, M. J., Che, S., et al. (2016). Autophagy Flux in CA1 Neurons of Alzheimer hippocampus: Increased Induction Overburdens Failing Lysosomes to Propel Neuritic Dystrophy. Autophagy 12, 2467-2483. doi:10.1080/15548627.2016.1239003

Bravo-San Pedro, J. M., Sica, V., Martins, I., Pol, J., Loos, F., Maiuri, M. C., et al. (2019). Acyl-CoA-binding Protein Is a Lipogenic Factor that Triggers Food Intake and Obesity. Cel. Metab. 37, 754-767. doi:10. 1016/j.cmet.2019.07.010

Breccia, M., Mascaritoli, M., Aversa, Z., Mandelli, F., and Alimena, G. (2005). Imatinib Mesylate May Improve Fasting Blood Glucose in Diabetic Ph+ Chronic Myelogenous Leukemia Patients Responsive to Treatment. J. Clin. Oncol. 15, 4653-4655. doi:10.1200/JCO.2004.04.217

Cai, J., Pires, K. M., Ferhat, M., Chaurasia, B., Buffolo, M. A., Smalling, R., et al. (2018). Autophagy Ablation in Adipocytes Induces Insulin Resistance and Reveals Roles for Lipid Peroxide and Nrf2 Signaling in Adipose-Liver Crosstalk. Cell Rep. 25, 1708-1717. doi:10.1016/j.celrep.2018.10.040

Campbell, G. R., and Spector, S. A. (2011). Hormonally Active Vitamin D3 (1alpha,25-Dihydroxycholecalciferol) Triggers Autophagy in Human Macrophages that Inhibits HIV-1 Infection. J. Biol. Chem. 286, 18890-18902. doi:10.1074/jbc.m110.206110

Can, G., Ekiz, H. A., and Baran, Y. (2013). Imatinib Induces Autophagy through BECLIN-1 and ATG5 Genes in Chronic Myeloid Leukemia Cells. Hematology 16, 95-99. doi:10.1179/102453311x12902908412039

Candeias, E., Sebastião, I., Cardoso, S., Carvalho, C., Santos, M. S., Oliveira, C. R., et al. (2018). Brain GLP-1/IGF-1 Signaling and Autophagy Mediate Exendin-4 protection against Apoptosis in Type 2 Diabetic Rats. Mol. Neurobiol. 55, 4030-4050. doi:10.1007/s12035-017-0622-3

Cao, Q., Du, H., Fu, X., Duan, N., Liu, C., and Li, X. (2019). Artemisinin Attenuated Atherosclerosis in High-Fat Diet-Fed ApoE-/- Mice by Promoting Macrophage Autophagy through the AMPK/mTOR/ULK1 Pathway. J. Cardiovas. Pharmacol. 75, 321-332. doi:10.1097/FJC.0000000000000794

Castillo, K., Nassif, M., Valenzuela, V., Rohas, F., Matus, S., Mercado, G., et al. (2013). Trehalose Delays the Progression of Amyotrophic Lateral Sclerosis by 
Enhancing Autophagy in Motoneurons. Autophagy 9, 1308-1320. doi:10.4161/ auto. 25188

Castoldi, F., Hyvönen, M. T., Durand, S., Aprahamian, F., Sauvat, A., Malik, S. A., et al. (2020). Chemical Activation of SAT1 Corrects Diet-Induced Metabolic Syndrome. Cell Death Differ. 27, 2904-2020. doi:10.1038/s41418-020-0550-z

Cen, X., Chen, Y., Xu, X., Wu, R., He, F., Zhao, Q., et al. (2020). Pharmacological Targeting of MCL-1 Promotes Mitophagy and Improves Disease Pathologies in an Alzheimer's Disease Mouse Model. Nat. Commun. 11, 5731. doi:10.1038/ s41467-020-19547-6

Chauhan, S., Ahmed, Z., Bradfute, S., Arko-Mensah, J., Mandell, M. A., Won, S. C., et al. (2015). Pharmaceutical Screen Identifies Novel Target Processes for Activation of Autophagy with a Broad Translational Potential. Nat. Commun. 6, 8620. doi:10.1038/ncomms 9620

Chen, C., Liu, Y., Liu, Y., and Zheng, P. (2009). mTOR Regulation and Therapeutic Rejuvenation of Aging Hematopoietic Stem Cells. Sci. Signal. 2, ra75. doi:10. 1126/scisignal.2000559

Chen, Z.-F., Li, Y.-B., Han, J.-Y., Yin, J.-J., Wang, Y., Zhu, L.-B., et al. (2013). Liraglutide Prevents High Glucose Level Induced Insulinoma Cells Apoptosis by Targeting Autophagy. Chin. Med. J. 126, 937-941.

Cheng, Y., Pan, X., Wang, J., Li, X., Yang, S., Yin, R., et al. (2020). Fucoidan Inhibits NLRP3 Inflammasome Activation by Enhancing p62/SQSTM1-dependent Selective Autophagy to Alleviate Atherosclerosis. Oxid. Med. Cel. Longev. 2020, 3186306. doi:10.1155/2020/3186306

Chude, C. I., and Amaravadi, R. K. (2017). Targeting Autophagy in Cancer: Update on Clinical Trials and Novel Inhibitors. Int. J. Mol. Sci. 18, 1279. doi:10.3390/ ijms18061279

Chung, C. Y., Shin, H. R., Berdan, C. A., Ford, B., Ward, C. C., Olzmann, J. A., et al. (2019). Covalent Targeting of the Vacuolar H +-ATPase Activates Autophagy via mTORC1 Inhibition. Nat. Chem. Biol. 15, 776-785. doi:10.1038/s41589019-0308-4

Cianfanelli, V., Fuoco, C., Lorente, M., Salazar, M., Quondamatteo, F., Gherardini, P. F., et al. (2015). AMBRA1 Links Autophagy to Cell Proliferation and Tumorigenesis by Promoting C-Myc Dephosphorylation and Degradation. Nat. Cel Biol. 17, 20-30. doi:10.1038/ncb3072

Cloherty, A. P. M., van Teijlingen, N. H., Eisden, T. T. H. D., van Hamme, J. L., Rader, A. G., Geijtenbeek, T. B. H., et al. (2021). Autophagy-enhancing Drugs Limit Mucosal HIV-1 Acquisition and Suppress Viral Replication Ex Vivo. Sci. Rep. 11, 4767. doi:10.1038/s41598-021-84081-4

Coupe, B., Ishii, Y., Dietrich, M. O., Komatsu, M., Horvath, T. L., and Bouret, S. G. (2012). Loss of Autophagy in Pro-opiomelanocortin Neurons Perturbs Axon Growth and Causes Metabolic Dysregulation. Cel. Metab. 15, 1-9. doi:10.1016/ j.cmet.2011.12.016

Dai, D.-F., Karunadharma, P. P., Chiao, Y. A., Basisty, N., Crispin, D., Hsieh, E. J., et al. (2014). Altered Proteome Turnover and Remodeling by Short-Term Caloric Restriction or Rapamycin Rejuvenate the Aging Heart. Aging Cell 13, 529-539. doi:10.1111/acel.12203

Darios, F., and Stevanin, G. (2020). Impairment of Lysosome Function and Autophagy in Rare Neurodegenerative Diseases. J. Mol. Biol. 432, 2714-2734. doi:10.1016/j.jmb.2020.02.033

DeBosch, B. J., Heitmeier, M. R., Mayer, A. L., Higgins, C. B., Crowley, J. R., Kraft, T. E., et al. (2016). Trehalose Inhibits Solute Carrier 2A (SLC2A) Proteins to Induce Autophagy and Prevent Hepatic Steatosis. Sci. Signal. 9, ra21. doi:10. 1126/scisignal.aac5472

Dehay, B., Bove, J., Rodriguez-Muela, N., Perier, C., Recasens, A., Boya, P., et al. (2010). Pathogenic Lysosomal Depletion in Parkin's Disease. Neurobiol. Dis. 30, 12534-12544. doi:10.1523/JNEUROSCI.1920-10.2010

Deter, R. L., and de Duve, C. (1967). Influence of Glucagon, an Induer of Cellular Autophagy, on Some Physical Properties of Rat Liver Lysosomes. J. Cel. Biol. 33, 437-449. doi:10.1083/jcb.33.2.437

Ding, Y., Fei, Y., and Lu, B. (2021). Emerging New Concepts of Degrader Technologies. Trends Pharmacol. Sci. 41, 464-474. doi:10.1016/j.tips.2020. 04.005

Doulatov, S., Vo, L. T., Macari, E. R., Wahlster, L., Kinney, M. A., Taylor, A. M., et al. (2017). Drug Discovery for Diamond-Blackfan Anemia Using Reprogrammed Hematopoietic Progenitors. Sci. Transl. Med. 9, 376. doi:10. 1126/scitranslmed.aah5645
Druker, B. J., Tamura, S., Buchdunger, E., Ohno, S., Segal, G. M., Fanning, S., et al. (1996). Effects of a Selective Inhibitor of the Abl Tyrosine Kinase on the Growth of Bcr-Abl Positive Cells. Nat. Med. 2, 561-566. doi:10.1038/nm0596-561

Du, J., Liang, Y., Xu, F., Sun, B., and Wang, Z. (2013). Trehalose Rescues Alzheimer's Disease Phenotypes in APP/PS1 Transgenic Mice. J. Pharm. Pharmacol. 65, 1753-1756. doi:10.1111/jphp.12108

Duensing, A., Medeiros, F., McConary, B., Josehp, N. E., Panigrahy, D., Singer, S., et al. (2004). Mechanism of Oncogenic KIT Signal Transduction in Primary Gastrointestinal Stromal Tumors (GISTs). Oncogene 23, 3999-4006. doi:10. 1038/sj.onc. 1207525

Ebato, C., Uchida, T., Arakawa, M., Komatsu, M., Ueno, T., Komiya, K., et al. (2008). Autophagy Is Important in Islet Homeostasis and Compensatory Increase of Beta Cell Mass in Response to High-Fat Diet. Cel. Metab. 8, 325-332. doi:10.1016/j.cmet.2008.08.009

Egan, D. F., Shackelford, D. B., Mihaylova, M. M., Gelino, S., Kohnz, R. A., Mair, W., et al. (2011). Phosphorylation of ULK1 (hATG1) by AMP-Activated Protein Kinase Connects Energy Sensing to Mitophagy. Science 331, 456-561. doi:10.1126/science.1196371

Eisenberg, T., Abdellatif, M., Schroeder, S., Primessnig, U., Stekovic, S., Pendl, T., et al. (2016). Cardioprotection and Lifespan Extension by the Natural Polyamine Spermidine. Nat. Med. 22, 1428-1438. doi:10.1038/nm.4222

Eisenberg, T., Knauer, H., Schauer, A., Büttner, S., Ruckenstuhl, C., CarmonaGutierrez, D., et al. (2009). Induction of Autophagy by Spermidine Promotes Longevity. Nat. Cel. Biol. 11, 1305-1314. doi:10.1038/ncb1975

Etmer, A., Huber, V., Gilch, S., Yoshimor, T., Erfle, V., Duyster, J., et al. (2007). The Anticancer Drug Imatinib Induces Cellular Autophagy. Leukemia 21, 936-942.

Fan, Y., Wang, N., Rocchi, A., Zhang, W., Vassar, R., Zhou, Y., et al. (2017). Identification of Natural Products with Neuronal and Metabolic Benefits through Autophagy Induction. Autophagy 13, 41-56. doi:10.1080/15548627.2016.1240855

Fang, Y., Ji, L., Zhu, C., Xiao, Y., Zhang, J., Lu, J., et al. (2020). Liraglutide Alleviates Hepatic Steatosis by Activating the TFEB-Regulated Autophagy-Lysosomal Pathway. Front. Cel. Dev. Biol. 8, 602574. doi:10.3389/fcell.2020.602574

Fernández, A. F., Bárcena, C., Martínez-García, G. G., Tamargo-Gómez, I., Suárez, M. F., Pietrocola, F., et al. (2017). Autophagy Couteracts Weight Gain, Lipotoxicity and Pancreatic $\beta$-cell Death upon Hypercaloric Pro-diabetic Regimens. Cell Death Dis. 8, e2970.

Fernando, R., Castro, J. P., Flore, T., Deubel, S., Grune, T., and Ott, C. (2020). Age-related Maintenance of the Autophagy-Lysosomal System Is Dependent on Skeletal Muscle Type. Oxid. Med. Cel. Longev. 2020, 4908162. doi:10.1155/2020/4908162

Floto, R. A., Sarkar, S., Perlstein, E. O., Kampmann, B., Schreiber, S. L., and Rubinsztein, D. C. (2008). Small Molecule Enhancers of Rapamycin-Induced TOR Inhibition Promote Autophagy, Reduce Toxicity in Huntington's Disease Models and Enhance Killing of Mycobacteria by Macrophages. Autophagy 3, 620-622. doi:10.4161/auto.4898

Gatica, D., Chiong, M., Lavandero, S., and J Klionsky, D. (2021). The Role of Autophagy in Cardiovascular Pathology. Cardiovasc. Res. in press. doi:10.1093/ cvr/crab158

Ghosh, S., Dellibovi-Ragheb, T. A., Kerviel, A., Pak, E., Qiu, Q., Fisher, M., et al. (2020). $\beta$-Coronaviruses Use Lysosomes for Egress Instead of the Biosynthetic Secretory Pathway. Cell 183, 1520-1535. doi:10.1016/j.cell.2020.10.039

Grégoire, I. P., Rabourdin-Combe, C., and Faure, M. (2011). Autophagy and RNA Virus Interactomes Reveal IRGM as a Common Target. Autophagy 8, 1137.

Gutierrez, M. G., Master, S. S., Singh, S. B., Taylor, G. A., Colombo, M. I., and Deretic, V. (2004). Autophagy Is a Defense Mechanism Inhibiting BCG and Mycobacterium tuberculosis Survival in Infected Macrophages. Cell 119, 753-766. doi:10.1016/j.cell.2004.11.038

Han, M. S., Chung, K. W., Cheon, H. G., Rhee, S. D., Yoon, C.-H., Lee, M-K. L., et al. (2009). Imatinib Mesylate Reduces Endoplasmic Reticulum Stress and Induces Remission of Diabetes in Db/db Mice. Diabetes 58, 329-336. doi:10. $2337 / \mathrm{db} 08-0080$

Hansen, M., Rubinsztein, D. C., and Walker, D. W. (2018). Autophagy as a Promoter of Longevity: Insights from Model Organisms. Nat. Rev. Mol. Cel. Biol. 19, 579-593. doi:10.1038/s41580-018-0033-y

Harrison, D. E., Strong, R., Sharp, Z. D., Nelson, J. F., Astle, C. M., Flurkey, K., et al. (2009). Rapamycin Fed Late in Life Extends Lifespan in Genetically Heterogeneous Mice. Nature 460, 392-395. doi:10.1038/nature08221 
Hartmann, J. T., Haap, M., Kopp, H. G., and Lipp, H. P. (2009). Tyrosine Kinase Inhibitors - a Review on Pharmacology, Metabolism and Side Effects. Curr. Drug Metab. 10, 470-481. doi:10.2174/138920009788897975

Hawley, S. A., Ford, R. J., Smith, B. K., Gowans, G. J., Mancini, S. J., Pitt, R. D., et al. (2016). The $\mathrm{Na}+$ /glucose Cotransporter Inhibitor Canagliflozin Activates AMPK by Inhibiting Mitochondrial Function and Increasing Cellular AMP Levels. Diabetes 65, 2784-2794. doi:10.2337/db16-0058

He, C., Bassik, M. C., Moresi, V., Sun, K., Wei, Y., Zou, Z., et al. (2012). Exerciseinduced BCL2-Regulated Autophagy Is Required for Muscle Glucose Homeostasis. Nature 481, 511-515. doi:10.1038/nature10758

Hebron, M. L., Lonskaya, I., and Moussa, C. E. (2013). Nilotinib Reverses Loss of Dopamine Neurons and Improves Motor Behavior via Autophagic Degradation of $\alpha$-synuclein in Parkinson's Disease Models. Hum. Mol. Genet. 22, 3315-3328. doi:10.1093/hmg/ddt192

Honda, Y., Tanaka, M., and Honda, S. (2010). Trehalose Extends Longevity in the Nematode Caenorhabditis elegans. Aging Cell 9, 558-569. doi:10.1111/j.14749726.2010.00582.x

Hoseini, A., Namazi, G., Farrokhian, A., Reiner, Ž., Aghadavod, E., Bahmani, F., et al. (2019). The Effects of Resveratrol on Metabolic Status in Patients with Type 2 Diabetes Mellitus and Coronary Heart Disease. Food Funct. 10, 6042-6051. doi:10.1039/c9fo01075k

Howell, G. M., Gomez, H., Collage, R. D., Loughran, P., Zhang, X., Escobar, D. A., et al. (2013). Augmenting Autophagy to Treat Acute Kidney Injury during Endotoxemia in Mice. PLOS ONE 8, e69520. doi:10.1371/journal. pone. 0069520

Howell, J. J., Hellberg, K., Turner, M., Talbott, G., Kolar, M. J., Ross, D. S., et al. (2017). Metformin Inhibits Hepatic mTORC1 Signaling via Dose-dependent Mechanisms Involving AMPK and the TSC Complex. Cel. Metab. 25, 463-471. doi:10.1016/j.cmet.2016.12.009

Hsiao, P.-J., Chiou, H.-Y. C., Jiang, H.-J., Lee, M.-Y., Hsieh, T.-J., and Kuo, K.-K. (2017). Pioglitazone Enhances Cytosolic Lipolysis, $\beta$-oxidation and Autophagy to Ameliorate Hepatic Steatosis. Sci. Rep. 7, 9030. doi:10.1038/s41598-01709702-3

Huab, W., Chanb, H., Lu, L., Wong, K. T., Wong, S. H., Sunny, T. W., et al. (2020). Autophagy in Intracellular Bacterial Infection. Sem. Cel. Dev. Biol. 101, 41-50. doi:10.1016/j.semcdb.2019.07.014

Hütter, E., Skovbro, M., Lener, B., Prats, C., Rabøl, R., Dela, F., et al. (2007). Oxidative Stress and Mitochondrial Impairment Can Be Separated from Lipofuscin Accumulation in Aged Human Skeletal Muscle. Aging Cell 6, 245-256. doi:10.1111/j.1474-9726.2007.00282.x

Jeong, S.-J., Stitham, J., Evans, T. D., Zhang, X., Rodriguez-Velez, A., Yeh, Y.-S., et al. (2021). Trehalose Causes Low-Grade Lysosomal Stress to Activate TFEB and the Autophagy-Lysosome Biogenesis Response. Autophagy 11, 1-13. doi:10.1080/15548627.2021.1896906

Ji, J., Xue, T. F., Guo, X. D., Yang, J., Guo, R. B., Wang, J., et al. (2018). Antagonizing Peroxisome Proliferator-Activated Receptor $\gamma$ Facilitates M1-To-M2 Shift of Microglia by Enhancing Autophagy via the LKB1-AMPK Signaling Pathway. Aging Cell 17, e12774. doi:10.1111/acel.12774

Jiang, Y., Huang, W., Wang, J., Xu, Z., He, J., Lin, X., et al. (2014). Metformin Plays a Dual Role in MIN6 Pancreatic B Cell Function through AMPK-dependent Autophagy. Int. J. Biochem. Cel. Biol. 10, 268-277. doi:10.7150/ijbs.7929

Jung, H. S., Chung, K. W., Kim, J. W., Kim, J., Komatsu, M., Tanaka, K., et al. (2008). Loss of Autophagy Diminishes Pancreatic B-Cell Mass and Function with Resultant Hyperglycemia. Cel. Metab. 8, 318-324. doi:10.1016/j.cmet. 2008.08.013

Kaizuka, T., Morishita, H., Hama, Y., Tsukamoto, S., Matsui, T., Toyota, Y., et al. (2016). An Autophagic Flux Probe that Releases an Internal Control. Mol. Cel. 64, 835-849. doi:10.1016/j.molcel.2016.09.037

Kalender, A., Selvaraj, A., Kim, S. Y., Gulati, P., Brûlé, S., Viollet, B., et al. (2010). Metformin, Independent of AMPK, Inhibits mTORC1 in a Rag GTPase-dependent Manner. Cel. Metab. 11, 390-401. doi:10.1016/j.cmet. 2010.03.014

Kang, Y. H., Cho, M. H., Kim, J. Y., Kwon, M. S., Peak, J. J., Kang, S. W., et al. (2016). Impaired Macrophage Autophagy Induces Systemic Insulin Resistance in Obesity. Autophagy 7, 35577-35591. doi:10.18632/oncotarget.9590

Kaushal, K. P., Chandrashekar, K., Juncos, L. A., and Shah, S. V. (2020). Autophagy Function and Regulation in Kidney Disease. Biomolecules 10, 100. doi:10.3390/ biom 10010100
Kaushik, S., Arias, E., Kwon, H., Lopez, N. M., Athonvarangkull, D., Sahu, S., et al. (2012). Loss of Autophagy in Hypothalamic POMC Neurons Impairs Lipolysis. EMBO Rep. 13, 258-265. doi:10.1038/embor.2011.260

Kaushik, S., Rodriguez-Navarro, J. A., Arias, E., Kiffin, R., Sahu, S., Schwartz, G. J., et al. (2011). Autophagy in Hypothalamic AgRP Neurons Regulates Food Intake and Energy Balance. Cel Metab 14, 173-183. doi:10.1016/j.cmet.2011. 06.008

Kersten, S., Seydoux, J., Peters, J. M., Gonzalez, F. J., Desvergne, B., and Wahli, W. (1999). Peroxisome Proliferator-Activated Receptor Alpha Mediates the Adaptive Response to Fastin. J. Clin. Invest. 103, 1489-1498. doi:10.1172/ jci6223

Kim, J., Cheon, H., Jeong, Y. T., Quan, Y., Kim, K. H., Cho, J. M., et al. (2014). Amyloidogenic Peptide Oligomer Accumulation in Autophagy-Deficient B-Cells Leads to Diabetes. J. Clin. Invest. 125, 3311-3324. doi:10.1172/jci69625

Kim, J., Kim, S. H., Kang, H., Lee, S., Park, S.-Y., Cho, Y., et al. (2021a). TFEBGDF15 axis Protects against Obesity and Insulin Resistance as a Lysosomal Stress Response. Nat. Metab. 3, 410-427. doi:10.1038/s42255-021-00368-w

Kim, J., Kim, Y. C., Fang, C., Russel, R. C., Kim, J. H., Fan, W., et al. (2013a). Differential Regulation of Distinct Vps34 Complex by AMPK in Nutrient Stress and Autophagy. Cell 152, 290-303. doi:10.1016/j.cell.2012.12.016

Kim, J., Kundu, M., Viollet, B., and Guan, K.-L. (2011). AMPK and mTOR Regulate Autophagy through Direct Phosphorylation of ULK1. Nat. Cel. Biol. 13, 132-141. doi:10.1038/ncb2152

Kim, J., Park, K., Kim, M. J., Lim, H., Kim, K. H., Kim, S.-W., et al. (2021b). An Autophagy Enhancer Ameliorates Diabetes of Human IAPP-Transgenic Mice through Clearance of Amyloidogenic Oligomer. Nat. Commun. 12, 183. doi:10. 1038/s41467-020-20454-Z

Kim, K. H., Jeong, Y. T., Oh, H., Kim, S. H., Cho, J. M., Kim, Y.-N., et al. (2013b). Autophagy Deficiency Leads to protection from Obesity and Insulin Resistance by Inducing Fgf21 as a Mitokine. Nat. Med. 19, 83-92. doi:10.1038/nm.3014

Kim, K. H., and Lee, M.-S. (2014). Autophagy-a Key Player in Cellular and Body Metabolism. Nat. Rev. Endocrinol. 10, 322-337. doi:10.1038/nrendo.2014.35

Kimmel, A. C., and White, E. (2017). Autophagy and Tumor Metabolism. Cel. Metab. 25, 1037-1043. doi:10.1016/j.cmet.2017.04.004

Kivinen, N. (2018). The Role of Autophagy in Age-Related Macular Degeneration. Acta Opthalmol. 96 (SA110), 1-50. doi:10.1111/aos.13753

Klionsky, D. J., and Emr, S. D. (2000). Autophagy as a Regulated Pathway of Cellular Degradation. Science 290, 1717-1721. doi:10.1126/science.290.5497. 1717

Kumar, M. J. V., Shah, D., Giridharan, M., Yadav, N., Manjithaya, R., and Clement, J. P. (2021). Spatiotemporal Analysis of Soluble Aggregates and Autophagy Markers in the R6/2 Mouse Model. Sci. Rep. 11, 96. doi:10.1038/s41598-02078850-w

Kuramoto, K., Wang, N., Fan, Y., Zhang, W., Schoenen, F. J., Frankowski, K. J., et al. (2016). Autophagy Activation by Novel Inducers Prevents BECN2Mediated Drug Tolerance to Cannabinoids. Autophagy 12, 1460-1471. doi:10.1080/15548627.2016.1187367

Lampert, M. A., and Gustafsson, A. B. (2018). Balancing Autophagy for a Healthy Heart. Curr. Opin. Physiol. 1, 21-26. doi:10.1016/j.cophys.2017.11.001

Laybutt, D. R., Preston, A. M., Akerfeldt, M. C., Kench, J. G., Busch, A. K., Biankin, A. V., et al. (2007). Endoplasmic Reticulum Stress Contributes to Beta Cell Apoptosis in Type 2 Diabetes. Diabetologia 50, 752-763. doi:10.1007/s00125006-0590-z

Lazarou, M., Sliter, D. A., Kane, L. A., Sarraf, S. A., Wang, C., Burman, J. L., et al. (2015). The Ubiquitin Kinase PINK1 Recruits Autophagy Receptors to Induce Mitophagy. Nature 524. doi:10.1038/nature14893

Lee, H.-Y., Kim, J., Quan, Y., Lee, J.-C., Kim, M.-S., Km, S., et al. (2016). Autophagy Deficiency in Myeloid Cells Increases Susceptibility to Obesity-Induced Diabetes and Experimental Colitis. Autophagy 12, 1390-1403. doi:10.1080/ 15548627.2016.1184799

Lee, J. M., Wagner, M., Xiao, R., Kim, K. H., Feng, D., Lazar, M. A., et al. (2014). Nutrient-sensing Nuclear Receptors Coordinate Autophagy. Nature 516, 112-115. doi:10.1038/nature13961

Lee, S., Kim, S., Park, Y. J., Yun, S. P., Kwon, S.-H., Kim, D., et al. (2018). The C-Abl Inhibitor, Radotinib HCl, Is Neuroprotective in a Preclinical Parkinson's Disease Mouse Model. Hum. Mol. Genet. 27, 2344-2356. doi:10.1093/hmg/ddy143

Levy, J. M. M., Towers, C. G., and Thorburn, A. (2017). Targeting Autophagy in Cancer. Nat. Rev. Cancer 17, 528-542. doi:10.1038/nrc.2017.53 
Li, H., Zhang, Q., Yang, X., and Wang, L. (2018). PPAR-gamma Agonist Rosiglitazone Reduces Autophagy and Promotes Functional Recovery in Experimental Traumaticspinal Cord Injury. Neurosci. Lett. 650, 89-96. doi:10.1016/j.neulet.2017.02.075

Li, L., Chen, J., ., Zhou, Y., Zhang, J., and Chen, L. (2021). Artesunate Alleviates Diabetic Retinopathy by Activating Autophagy via the Regulation of AMPK/ SIRT1 Pathway. Arch. Physiol. Biochem. 4, 1-8. doi:10.1080/13813455.2021. 1887266

Li, Z., Wang, C., Wang, Z., Zhu, C., Li, J., Sha, T., et al. (2019). Allele-selective Lowering of Mutant HTT Protein by HTT-LC3 Linker Compounds. Nature 575, 203-209. doi:10.1038/s41586-019-1722-1

Liang, C., Feng, P., Ku, B., Dotan, I., Canaani, D., Oh, B. H., et al. (2006). Autophagic and Tumour Suppressor Activity of a Novel Beclin1-Binding Protein UVRAG. Nat. Cel. Biol. 8, 688-699. doi:10.1038/ncb1426

Liang, Y. T., Piao, C., Beuschel, C. B., Toppe, D., Kollipara, L., Bogdanow, B., et al. (2021). eIF5A Hypusination, Boosted by Dietary Spermidine, Protects from Premature Brain Aging and Mitochondrial Dysfunction. Cel. Rep. 35, 108941. doi:10.1016/j.celrep.2021.108941

Liao, X., Sluimer, J. C., Wang, Y., Subramanian, M., Brown, K., Pattison, J. S., et al. (2012). Macrophage Autophagy Plays a Protective Role in Advanced Atherosclerosis. Cel. Metab. 15, 545-553. doi:10.1016/j.cmet.2012.01.022

Lim, H., Lim, Y.-M., Kim, K. H., Jeon, Y. E., Park, K., Kim, J., et al. (2018). A Novel Autophagy Enhancer as a Therapeutic Agent against Metabolic Syndrome and Diabetes. Nat. Commun. 9, 1438. doi:10.1038/s41467-018-03939-w

Lim, S. W., Shin, Y. J., Luo, K., Quan, Y., Ko, E. J., Chung, B. H., et al. (2019). Effect of Klotho on Autophagy Clearance in Tacrolimus-Induced Renal Injury. FASEB J. 33, 2694-2706. doi:10.1096/fj.201800751r

Lim, Y.-M., Lim, H.-J., Hur, K. Y., Quan, W., Lee, H.-Y., Cheon, H., et al. (2014). Systemic Autophagy Insufficiency Compromises Adaptation to Metabolic Stress and Facilitates Progression from Obesity to Diabetes. Nat. Commun. 5, 4934. doi:10.1038/ncomms5934

Lin, F., Ghislat, G., Luo, S., Renna, M., Siddiqi, F., and Rubinsztein, D. C. (2015). XIAP and cIAP1 Amplifications Induce Beclin 1-dependent Autophagy through NFkB Activation. Hum. Mol. Genet. 24, 2899-2913. doi:10.1093/ $\mathrm{hmg} / \mathrm{ddv} 052$

Lin, Y.-C., Chang, P.-F., Lin, H.-F., Liu, K., Chang, M.-H., and Ni, Y.-H. (2016). Variants in the Autophagy-Related Gene IRGM Confer Susceptibility to Nonalcoholic Fatty Liver Disease by Modulating Lipophagy. J. Hepatol. 65, 1209-1216. doi:10.1016/j.jhep.2016.06.029

Liu, M., Xu, L., Yin, L., Qi, Y., Xu, Y., Han, X., et al. (2015a). Potent Effects of Dioscin against Obesity in Mice. Sci. Rep. 5, 7973. doi:10.1038/srep07973

Liu, W. J., Shen, T. T., Chen, R. H., Wu, H.-L., Wang, W. J., Deng, J. K., et al. (2015b). Autophagy-lysosome Pathway in Renal Tubular Epithelial Cells Is Disrupted by Advanced Glycation End Products in Diabetic Nephropathy. J. Biol. Chem. 290, 20499-20510. doi:10.1074/jbc.m115.666354

Liu, Y., Lear, T., Larsen, M., Lin, B., Cao, Q., Alfaras, I., et al. (2021). Modulation of Lysosomal Function as a Therapeutic Approach for Coronaviral Infections. Res. Sq. Preprint.

Liu, Y., Shoji-Kawata, S., Sumpter, R. M. J., Wei, Y., Ginet, V., Zhang, L., et al. (2013). Autosis Is a Na+,K+-ATPase-regulated Form of Cell Death Triggered by Autophagy-Inducing Peptides, Starvation, and Hypoxia-Ischemia. Proc. Natl. Acad. Sci. USA 110, 20364-20371. doi:10.1073/pnas.1319661110

Lotfi, P., Tse, D. Y., Di Ronza, A., Seymour, M. L., Martano, G., Cooper, J. D., et al. (2018). Trehalose Reduces Retinal Degeneration, Neuroinflammation and Storage burden Caused by a Lysosomal Hydrolase Deficiency. Autophagy 14, 1419-1434. doi:10.1080/15548627.2018.1474313

Luo, R., Su, L.-Y., Li, G., Yang, J., Liu, Q., Yang, L.-X., et al. (2020). Activation of PPARA-Mediated Autophagy Reduces Alzheimer Disease-like Pathology and Cognitive Decline in a Murine Model. Autophagy 16, 52-69. doi:10.1080/ 15548627.2019.1596488

Luu, J., and Palczewski, K. (2018). Human Aging and Disease: Lessons from AgeRelated Macular Degeneration. Proc. Natl. Acad. Sci. USA 115, 2866-2872. doi:10.1073/pnas.1721033115

Ma, L., Ni, Y., Hu, L., Zhao, Y., Zheng, L., Yang, S., et al. (2021). Spermidine Ameliorates High-Fat Diet-Induced Hepatic Steatosis and Adipose Tissue Inflammation in Preexisting Obese Mice. Life Sci. 265, 118739. doi:10.1016/ j.lfs. 2020.118739
Ma, X., Chen, Z., Wang, L., Wang, G., Wang, Z., Dong, X., et al. (2018). The Pathogenesis of Diabetes Mellitus by Oxidative Stress and Inflammation: Its Inhibition by Berberine. Front. Pharmacol. 9, 782. doi:10.3389/fphar.2018. 00782

Madeo, F., Eisenberg, T., Pietrocola, F., and Kroemer, G. (2018). Spermidine Health Dis. 359, eaan2788. doi:10.1126/science.aan 2788

Mariño, G., Pietrocola, F., Eisenberg, T., Kong, Y., Malik, S. A., Andryushkova, A., et al. (2014). Regulation of Autophagy by Cytosolic Acetyl-Coenzyme A. Mol. Cel. 53, 710-725. doi:10.1016/j.molcel.2014.01.016

Marselli, L., Bugliani, M., Suleiman, M., Olimpico, F., Masini, M., Petrini, M., et al. (2013). Beta-Cell Inflammation in Human Type 2 Diabetes and the Role of Autophagy. Diabetes Obes. Metab. 15 (S3), 130-136. doi:10.1111/dom.12152

Martin, K. R., Celano, S. L., Solitro, A. R., Gunaydin, H., Scott, M., O'Hagan, R. C., et al. (2018). A Potent and Selective ULK1 Inhibitor Suppresses Autophagy and Sensitizes Cancer Cells to Nutrient Stress. iScience 8, 74-84. doi:10.1016/j.isci. 2018.09.012

Masini, M., Bugliani, M., Lupi, R., del Guerra, S., Boggi, U., Filipponi, F., et al. (2009). Autophagy in Human Type 2 Diabetes Pancreatic Beta Cells. Diabetologia 52, 1083-1086. doi:10.1007/s00125-009-1347-2

Melendez, A., Talloczy, Z., Seaman, M., Eskelinen, E.-L., Hall, D. H., and Levine, B. (2003). Autophagy Genes Are Essential for Dauer Development and Life-Span Extension in C. elegans. Science 301, 1387-1391. doi:10.1126/science.1087782

Menzies, F. M., Garcia-Arencibia, M., Imarisio, S., O'Sullivan, N. C., Ricketts, T., Kent, B. A., et al. (2015). Calpain Inhibition Mediates Autophagy-dependent protection against Polyglutamine Toxicity. Cel. Death Differ. 22, 433-444. doi:10.1038/cdd.2014.151

Michiels, C. F., Kurdi, A., Timmermans, J.-P., De Meyer, G. R. Y., and Martinet, W. (2016). Spermidine Reduces Lipid Accumulation and Necrotic Core Formation in Atherosclerotic Plaques via Induction of Autophagy. Atherosclerosis 251, 319-327. doi:10.1016/j.atherosclerosis.2016.07.899

Miller, D. R., and Thorburn, A. (2021). Autophagy and Organelle Homeostasis in Cancer. Dev. Cel. in press. doi:10.1016/j.devcel.2021.02.010

Misawa, T., Takahama, M., Kozaki, T., Lee, H., Zou, J., Saitoh, T., et al. (2013). Microtubule-driven Spatial Arrangement of Mitochondria Promotes Activation of the NLRP3 Inflammasome. Nat. Immunol. 14, 454-460. doi:10.1038/ni.2550

Mizushima, N., and Komatsu, M. (2011). Autophagy: Renovation of Cells and Tissues. Cell 147, 728-741. doi:10.1016/j.cell.2011.10.026

Morselli, E., Mariño, G., Bennetzen, M. V., Eisenberg, T., Megalou, E., Schroeder, S., et al. (2011). Spermidine and Resveratrol Induce Autophagy by Distinct Pathways Converging on the Acetylproteome. J. Cel. Biol. 192, 615-629. doi:10. 1083/jcb.201008167

Nah, J., Zhai, P., Huang, C.-Y., Fernández, A. F., Mareedu, S., Levine, B., et al. (2020). Upregulation of Rubicon Promotes Autosis during Myocardial Ischemia/reperfusion Injury. J. Clin. Invest. 130, 2978-2991. doi:10.1172/ jcil32366

Nakagawa, I., Amano, A., Mizushima, N., Yamamoto, A., Yamaguchi, H., Kamimoto, T., et al. (2004). Autophagy Defends Cells against Invading Group A Streptococcus. Science 306, 1037-1040. doi:10.1126/science.1103966

Nam, S. A., Kim, W. Y., Kim, J. W., Park, S. H., Kim, H. L., Lee, M.-S., et al. (2019). Autophagy Attenuates Tubulointerstital Fibrosis through Regulating Transforming Growth Factor- $\beta$ and NLRP3 Inflammasome Signaling Pathway. Cel. Death Dis. 10, 78. doi:10.1038/s41419-019-1356-0

Narendra, D., Tanaka, A., Suen, D. F., and Youle, R. J. (2008). Parkin Is Recruited Selectively to Impaired Mitochondria and Promotes Their Autophagy. J. Cel. Biol. 183, 795-803. doi:10.1083/jcb.200809125

Nassour, J., Radford, R., Correia, A., Fusté, J. M., Schoell, B., Jauch, A., et al. (2019). Autophagic Cell Death Restricts Chromosomal Instability during Replicative Crisis. Nature 565, 659-663. doi:10.1038/s41586-019-0885-0

Neff, F., Flores-Dominguez, D., Ryan, D. P., Horsch, M., Schröder, S., Adler, T., et al. (2013). Rapamycin Extends Murine Lifespan but Has Limited Effects on Aging. J. Clin. Invest. 123, 3272-3291. doi:10.1172/jci67674

Nishimura, K., Shiima, R., Kashiwagi, K., and Igarashi, K. (2006). Decrease in Polyamines with Aging and Their Ingestion from Food and Drink. J. Biochem. 139, 81-90. doi:10.1093/jb/mvj003

Nixon, R. A. (2007). Autophagy, Amyloidogenesis and Alzheimer Disease. J. Cel. Sci. 120, 4081-4091. doi:10.1242/jcs.019265 
Nogueira-Recalde, U., Lorenzo-Gómeza, I., Blancoa, F. J., Lozab, M. I., Grassic, D., Shirinsky, V., et al. (2019). Fibrates as Drugs with Senolytic and Autophagic Activity for Osteoarthritis Therapy. EBioMedicine 45. doi:10.1016/j.ebiom. 2019.06.049

Noyan-Ashraf, M. H., Shikatani, E. A., Schuiki, I., Mukovozov, I., Wu, J., Li, R. K., et al. (2013). A Glucagon-like Peptide-1 Analog Reverses the Molecular Pathology and Cardiac Dysfunction of a Mouse Model of Obesity. Circulation 127, 74-85. doi:10.1161/circulationaha.112.091215

Okamoto, T., Ozawa, Y., Kamoshita, M., Osada, H., Toda, E., Kurihara, T., et al. (2016). The Neuroprotective Effect of Rapamycin as a Modulator of the mTOR$\mathrm{NF}-\mathrm{Kb}$ axis during Retinal Inflammation. PLOS ONE 11, e0146517. doi:10. 1371/journal.pone. 0146517

Orphanos, G. S., Ioannidis, G. N., and Ardavanis, A. G. (2009). Cardiotoxicity Induced by Tyrosine Kinase Inhibitors. Acta Oncol. 48, 964-970. doi:10.1080/ 02841860903229124

Owen, M. R., Doran, E., and Halestrap, A. P. (2000). Evidence that Metformin Exerts its Anti-diabetic Effects through Inhibition of Complex I of the Mitochondrial Respiratory Chain. Biochem. J. 348, 607-614. doi:10.1042/ bj3480607

Ozcan, U., Cao, Q., Yilmaz, E., Lee, A.-H., Iwakoshi, N. N., Ozdelen, E., et al. (2004). Endoplasmic Reticulum Stress Links Obesity, Insulin Action, and Type 2 Diabetes. Science 306, 457-461. doi:10.1126/science.1103160

Packer, M. (2020a). Autophagy-dependent and -independent Modulation of Oxidative and Organellar Stress in the Diabetic Heart by Glucose-Lowering Drugs. Cardiovasc. Diabetol. 19, 62. doi:10.1186/s12933-020-01041-4

Packer, M. (2020b). Mitigation of the Adverse Consequences of Nutrient Excess on the Kidney: A Unified Hypothesis to Explain the Renoprotective Effects of Sodium-Glucose Cotransporter 2 Inhibitors. Am. J. Nephrol. 51, 289-293. doi:10.1159/000506534

Palm, W., Park, Y., Wright, K., Pavlova, N. N., Tuveson, D. A., and Thompson, C. B. (2015). The Utilization of Extracellular Proteins as Nutrients Is Suppressed by mTORC1. Cell 162, 259-270. doi:10.1016/j.cell.2015.06.017

Papp, D., Kovács, T., Billes, V., Varga, M., Tarnóci, A., Hackler, L., et al. (2016). AUTEN-67, an Autophagy-Enhancing Drug Candidate with Potent Antiaging and Neuroprotective Effects. Autophagy 12, 273-286. doi:10.1080/15548627. 2015.1082023

Park, H., Kang, J.-H., and Lee, S. (2020). Autophagy in Neurodegenerative Diseases: a hunter for Aggregates. Iint. J. Mol. Sci. 21, 3369. doi:10.3390/ ijms21093369

Petersen, K. F., Dufour, S., Befroy, D., Garcia, R., and Shulman, G. I. (2004). Impaired Mitochondrial Activity in the Insulin-Resistant Offspring of Patients with Type 2 Diabetes. N. Engl. J. Med. 350, 664-671. doi:10.1056/nejmoa031314

Pfeifer, U. (1978). Inhibition by Insulin of the Formation of Autophagic Vacuoles in Rat Liver. J. Cel. Biol. 78, 152-167. doi:10.1083/jcb.78.1.152

Pietrocola, F., Lachkar, S., Enot, D. P., Niso-Santano, M., Bravo-San Pedro, J. M., Sica, V., et al. (2015). Spermidine Induces Autophagy by Inhibiting the Acetyltransferase EP300. Cel. Death Differ. 22, 509-516. doi:10.1038/cdd. 2014.215

Poillet-Perez, L., Sarry, J.-E., and Joffre, C. (2021). Autophagy Is a Major Metabolic Regulator Involved in Cancer Therapy Resistance. Cel. Rep. 36, 109528. doi:10. 1016/j.celrep.2021.109528

Pyo, J. O., Yoo, S. M., Ahn, H. H., Nah, J., Hong, S. H., Kam, T. I., et al. (2013). Overexpression of Atg5 in Mice Activates Autophagy and Extends Lifespan. Nat. Commun. 4, 2300. doi:10.1038/ncomms3300

Qu, X., Yu, J., Bhagat, G., Furuya, N., Hibshoosh, H., Troxel, A., et al. (2003). Promotion of Tumorigenesis by Heterozygous Disruption of the Beclin 1 Autophagy Gene. J. Clin. Invest. 112, 1809-1820. doi:10.1172/jci20039

Quan, W., Hur, K. Y., Lim, Y., Oh, S. H., Lee, J.-C., Kim, H. C., et al. (2012a). Autophagy Deficiency in Beta Cells Leads to Compromised Unfolded Protein Response and Progression from Obesity to Diabetes in Mice. Diabetologia 55, 392-403. doi:10.1007/s00125-011-2350-y

Quan, W., Kim, H.-K., Moon, E.-Y., Kim, S. S., Choi, C. S., Komatsu, M., et al. (2012b). Role of Hypothalamic Proopiomelanocortin Neuron Autophagy in the Control of Appetite and Leptin Response. Endocrinology 153, 1817-1826. doi:10.1210/en.2011-1882

Rahman, M. A., and Rhim, H. (2017). Therapeutic Implication of Autophagy in Neurodegenerative Diseases. BMP Rep. 50, 345-354. doi:10.5483/bmbrep.2017. 50.7 .069
Ranek, M. J., Kokkonen-Simon, K. M., Chen, A., Dunkerly-Eyring, B. L., Vera, M. P., Oeing, C. U., et al. (2019). PKG1-modified TSC2 Regulates mTORC1 Activity to Counter Adverse Cardiac Stress. Nature 566, 264-269. doi:10. 1038/s41586-019-0895-y

Ren, H., Shao, Y., Wu, C., Ma, X., Lv, C., and Wang, Q. (2019). Metformin Alleviates Oxidative Stress and Enhances Autophagy in Diabetic Kidney Disease via AMPK/SIRT1-FoxO1 Pathway. Mol. Cel. Endocrino. 600, 110628. doi:10.1016/j.mce.2019.110628

Rivera, J. F., Costes, S., Gurlo, T., Glabe, C., and Butler, P. C. (2014). Autophagy Defends Pancreatic B-Cells from Human Islet Amyloid Polypeptide-Induced Toxicity. J. Clin. Invest. 124, 3489-3500. doi:10.1172/jci71981

Rocchi, A., Yamamoto, S., Ting, T., Fan, Y., Sadleir, K., Wang, Y., et al. (2017). A Becn1 Mutation Mediates Hyperactive Autophagic Sequestration of Amyloid Oligomers and Improved Cognition in Alzheimer's Disease. PLOS Genet. 13, e1006962. doi:10.1371/journal.pgen.1006962

Rose, C., Menzies, F. M., Renna, M., Acevedo-Arozena, A., Corrochano, S., Sadiq, O., et al. (2010). Rilmenidine Attenuates Toxicity of Polyglutamine Expansions in a Mouse Model of Huntington's Disease. Hum. Mol. Genet. 19, 2144-2153. doi:10.1093/hmg/ddq093

Rubinsztein, D. C., Codogno, P., and Levine, B. (2012). Autophagy Modulation as a Potential Therapeutic Target for Diverse Diseases. Nat. Rev. Drug Discov. 11, 709-730. doi:10.1038/nrd3802

Rubinsztein, D. C. (2006). The Roles of Intracellular Protein-Degradation Pathways in Neurodegeneration. Nature 443, 780-786. doi:10.1038/ nature05291

Salminen, A., and Kaarniranta, K. (2009). Regulation of the Aging Process by Autophagy. Trends Mol. Med. 15, 217-224. doi:10.1016/j.molmed.2009.03.004

Sarbassov, D. D., Ali, S. M., and Sabatini, D. M. (2005a). Growing Roles for the mTOR Pathway. Curr. Opin. Cel. Biol. 17, 596-603. doi:10.1016/j.ceb.2005. 09.009

Sarbassov, D. D., Guertin, D. A., Ali, S. M., and Sabatini, D. M. (2005b). Phosphorylation and Regulation of Akt/PKB by the Rictor-mTOR Complex. Science 307, 1098-1101. doi:10.1126/science.1106148

Sarbessov, D. D., Ali, S. M., Sengupta, S., Sheen, J.-H., Hsu, P. P., Bagley, A. F., et al. (2006). Prolonged Rapamycin Treatment Inhibits mTORC2 Assembly and Akt/PKB. Mol. Cel. 22, 159-168. doi:10.1016/j.molcel.2006.03.029

Sarkar, S., Davies, J. E., Huang, Z., Tunnacliffe, A., and Rubinsztein, D. C. (2007a). Trehalose, a Novel mTOR-independent Autophagy Enhancer, Accelerates the Clearance of Mutant Huntingtin and A-Synuclein. J. Biol. Chem. 282, 5641-5652. doi:10.1074/jbc.m609532200

Sarkar, S., Floto, R. A., Berger, Z., Imarisio, S., Cordenier, A., Pasco, M., et al. (2005). Lithium Induces Autophagy by Inhibiting Inositol Monophosphatase. J. Cel. Biol. 170, 1101-1111. doi:10.1083/jcb.200504035

Sarkar, S., Perlstein, E. O., Imarisio, S., Pineau, S., Cordenier, A., Maglathlin, R. L., et al. (2007b). Small Molecules Enhance Autophagy and Reduce Toxicity in Huntington's Disease Models. Nat. Chem. Biol. 3, 331-338. doi:10.1038/ nchembio883

Sataranatarajan, K., Ikeno, Y., Bokov, A., Feliers, D., Yalamanchili, H., Lee, H. J., et al. (2016). Rapamycin Increases Mortality in Db/db Mice, a Mouse Model of Type 2 Diabetes. J. Gerontol. 71, 850-857. doi:10.1093/gerona/glv170

Schroeder, S., Hofer, S. J., Zimmermann, A., Pechlaner, R., Dammbrueck, C., Pendl, T., et al. (2021). Dietary Spermidine Improves Cognitive Function. Cel. Rep. 35, 108985. doi:10.1016/j.celrep.2021.108985

Sciarretta, S., Zhai, P., Shao, D., Maejima, Y., Robbins, J., Volpe, M., et al. (2012). Rheb Is a Critical Regulator of Autophagy during Myocardial Ischemia: Pathophysiological Implications in Obesity and Metabolic Syndrome. Circulation 125, 1134-1146. doi:10.1161/circulationaha.111. 078212

Sergin, I., Evans, T. D., Zhang, X., Bhattacharya, S., Stokes, C. J., Song, E., et al. (2017). Exploiting Macrophage Autophagy-Lysosomal Biogenesis as a Therapy for Atherosclerosis. Nat. Commun. 8, 15750. doi:10.1038/ncomms15750

Settembre, C., Di Malta, C., Polito, V. A., Arencibia, M. G., Vetrini, F., Erdin, S., et al. (2011). TFEB Links Autophagy to Lysosomal Biogenesis. Science 332, 1429-1433. doi:10.1126/science.1204592

Sharma, S., Mells, J. E., Fu, P. P., Saxena, N. K., and Anania, F. A. (2011). GLP-1 Analogs Reduce Hepatocyte Steatosis and Improve Survival by Enhancing the Unfolded Protein Response and Promoting Macroautophagy. PLOS ONE 6, e25269. doi:10.1371/journal.pone.0025269 
Sheen, J. H., Zoncu, R., Kim, D., and Sabatini, D. M. (2011). Defective Regulation of Autophagy upon Leucine Deprivation Reveals a Targetable Liability of Human Melanoma Cells In Vitro and In Vivo. Cancer Cell 19, 613-628. doi:10.1016/j. ccr.2011.03.012

Shen, D., Wang, X., Li, X., Zhang, X., Yao, Z., Dibble, S., et al. (2012). Lipid Storage Disorders Block Lysosomal Trafficking by Inhibiting a TRP Channel and Lysosomal Calcium Release. Nat. Commun. 3, 731. doi:10.1038/ncomms1735

Shigihara, N., Fukunaka, A., Hara, A., Komiya, K., Honda, A., Uchida, T., et al. (2014). Human IAPP-Induced Pancreatic Beta-Cell Toxicity and its Regulation by Autophagy. J. Clin. Invest. 124, 3634-3644. doi:10.1172/jci69866

Shin, N.-R., Lee, J.-C., Lee, Y.-Y., Kim, M.-S., Whon, T. W., Lee, M.-S., et al. (2014). An Increase in the Akkermansia Sp. Population Induced by Metformin Treatment Improves Glucose Homeostasis in Diet-Induced Obese Mice. Gut 63, 727-735. doi:10.1136/gutjnl-2012-303839

Shirakabe, A., Zhai, P., Ikeda, Y., Saito, T., Maejima, Y., Hsu, C. P., et al. (2016). Drp1-dependent Mitochondrial Autophagy Plays a Protective Role against Pressure Overload-Induced Mitochondrial Dysfunction and Heart Failure. Circulation 133, 1249-1263. doi:10.1161/circulationaha.115.020502

Shoji-Kawata, S., Sumpter, R., Leveno, M., Campbell, G. R., Zou, Z., Kinch, L., et al. (2013). Identification of a Candidate Therapeutic Autophagy-Inducing Peptide. Nature 494, 201-206. doi:10.1038/nature11866

Siddiqi, F. H., Menzies, F. M., Lopez, A., Stamatakou, E., Karabiyik, C., Ureshino, R., et al. (2019). Felodipine Induces Autophagy in Mouse Brains with Pharmacokinetics Amenable to Repurposing. Nat. Commun. 10, 1817. doi:10.1038/s41467-019-09494-2

Simuni, T., Fiske, B., Merchant, K., Coffey, C. S., Klingner, E., Caspell-Garcia, C., et al. (2021). Efficacy of Nilotinib in Patients with Moderately Advanced Parkinson Disease: a Randomized Clinical Trial. JAMA Neurol. 78, 312-320. doi:10.1001/jamaneurol.2020.4725

Singh, R., Kaushik, S., Wang, Y., Xiang, Y., Novak, I., Komatsu, M., et al. (2009a). Autophagy Regulates Lipid Metabolism. Nature 458, 1131-1135. doi:10.1038/ nature07976

Singh, R., Xiang, Y., Wang, Y., Baikati, K., Cuervo, A. M., Luu, Y. K., et al. (2009b). Autophagy Regulates Adipose Mass and Differentiation in Mice. J. Clin. Invest. 119, 3329-3339. doi:10.1172/JCI39228

Sohn, M., Kim, K., Uddin, M. J., Lee, G., Hwang, I., Kang, H., et al. (2017). Delayed Treatment with Fenofibrate Protects against High-Fat Diet-Induced Kidney Injury in Mice: the Possible Role of AMPK Autophagy. Am. J. Physiol. 312, F323-F334. doi:10.1152/ajprenal.00596.2015

Son, Y., Cho, Y. K., Saha, A., Kwon, H. J., Park, J.-H., Kim, M., et al. (2020). Adipocyte-specific Beclin1 Deletion Impairs Lipolysis and Mitochondrial Integrity in Adipose Tissue. Mol. Metab. 39, 101005. doi:10.1016/j.molmet. 2020.101005

Song, W., Wang, F., Lotfi, P., Sardiello, M., and Segatori, L. (2014). 2Hydroxypropyl- $\beta$-cyclodextrin Promotes Transcription Factor EB-Mediated Activation of Autophagy: Implications for Therapy. J. Biol. Chem. 289. doi:10. 1074/jbc.M113.506246

Song, Y. M., Lee, Y.-h., Kim, J.-W., Ham, D.-S., Kang, E.-S., Cha, B. S., et al. (2015). Metformin Alleviates Hepatosteatosis by Restoring SIRT1-Mediated Autophagy Induction via an AMP-Activated Protein Kinase-independent Pathway. Autophagy 11, 46-59. doi:10.4161/15548627.2014.984271

Spiegelman, B. M. (1998). PPAR-gamma: Adipogenic Regulator and Thiazolidinedione Receptor. Diabetes 47, 507-514. doi:10.2337/diabetes.47. 4.507

Steele, J. W., and Gandy, S. (2013). Latrepirdine (Dimebon), a Potential Alzheimer Therapeutic, Regulates Autophagy and Neuropathology in an Alzheimer Mouse Model. Autophagy 9, 617-618. doi:10.4161/auto.23487

Sun, N., Yun, J., Liu, J., Malide, D., Liu, C., Rovira, I. I., et al. (2015). Measuring In Vivo Mitophagy. Mol. Cel 60, 685-696. doi:10.1016/j.molcel.2015.10.009

Sun, Y., Xia, M., Yan, H., Han, Y., Zhang, F., Hu, Z., et al. (2018). Berberine Attenuates Hepatic Steatosis and Enhances Energy Expenditure in Mice by Inducing Autophagy and Fibroblast Growth Factor 21. Br. J. Pharma. 175, 374-387. doi:10.1111/bph.14079

Suresh, S. N., Chavalmane, A. K., Dj, V., Yarreiphang, H., Rai, S., Paul, A., et al. (2017). A Novel Autophagy Modulator 6-Bio Ameliorates SNCA/alphasynuclein Toxicity. Autophagy 13, 1221-1234. doi:10.1080/15548627.2017. 1302045
Suresh, S. N., Chavalmane, A. K., Pillai, M., Ammanathan, V., Vidyadhara, D. J., Yarreiphang, H., et al. (2018). Modulation of Autophagy by a Small Molecule Inverse Agonist of ERRa Is Neuroprotective. Front. Mol. Neurosci. 11, 109. doi:10.3389/fnmol.2018.00109

Takahashi, D., Moriyama, J., Nakamura, T., Miki, E., Takahashi, E., Sato, A., et al. (2019). AUTACs: Cargo-specific Degraders Using Selective Autophagy. Mol. Cel. 76, 797-810. doi:10.1016/j.molcel.2019.09.009

Tang, C., Livingston, M. J., Liu, Z., and Dong, Z. (2020). Autophagy in Kidney Homeostasis and Disease. Nat. Rev. Nephrol. 16, 489-508. doi:10.1038/s41581020-0309-2

Tedeschi, V., Petrozziello, T., Sisalli, M. S., Boscia, F., Canzoniero, L. M. T., and Secondo, A. (2019). The Activation of Mucolipin TRP Channel 1 (TRPML1) Protects Motor Neurons from L-BMAA Neurotoxicity by Promoting Autophagic Clearance. Sci. Rep. 9, 10743. doi:10.1038/s41598-019-46708-5

Thelen, M., and Brown-Borg, H. M. (2020). Does Diet Have a Role in the Treatment of Alzheimer's Disease? Front. Aging Neurosci. 12, 617071. doi:10.3389/fnagi.2020.617071

Tian, Y., Bustos, V., Flajolet, M., and Greengard, P. (2011). A Small-Molecule Enhancer of Autophagy Decreases Level of Abeta and APP-CTF via Atg5dependent Autophagy Pathway. FASEB J. 25, 1934-1942. doi:10.1096/fj.10175158

Torra, P., Gervois, P., and Staels, B. (2000). Peroxisome Proliferator-Activated Receptor Alpha in Metabolic Disease, Inflammation, Atherosclerosis and Aging. Curr. Opin. Lipidol. 10, 151-159. doi:10.1097/00041433-19990400000009

Tsunemi, T., Perez-Rosello, T., Ishiguro, Y., Yoroisaka, A., Jeon, S., Hamada, K., et al. (2019). Increased Lysosomal Exocytosis Induced by Lysosomal $\mathrm{Ca}(2+)$ Channel Agonists Protects Human Dopaminergic Neurons from AlphaSynuclein Toxicity. J. Neurosci. 39, 5760-5772. doi:10.1523/jneurosci.308518.2019

Uddin, M. N., Elahi, M., Shimonaka, S., Kakuta, S., Ishiguro, K., Motoi, Y., et al. (2021). Strain-specific Clearance of Seed-dependent Tau Aggregation by Lithium-Induced Autophagy. Biochem. Biophys. Res. Com. 543, 65-71. doi:10.1016/j.bbrc.2020.12.113

Veneri, D., Rranchini, M., and Bonora, E. (2005). Imatinib and Regression of Type 2 Diabetes. N. Engl. J. Med. 352, 1049-1050. doi:10.1056/ nejm200503103521023

Wada, S., Kubota, Y., Sawa, R., Umekita, M., Hatano, M., Ohba, S., et al. (2015). Novel Autophagy Inducers Lentztrehaloses A, B and C. J. Antibiot. 68, 521-529. doi:10.1038/ja.2015.23

Wang, C., Niederstrasser, H., Douglas, P. M., Lin, R., Jaramillo, J., Li, Y., et al. (2017). Small-molecule TFEB Pathway Agonists that Ameliorate Metabolic Syndrome in Mice and Extend C. elegans Lifespan. Nat. Commun. 8, 2270. doi:10.1038/s41467-017-02332-3

Wang, J., Shen, L., Hong, H., Li, J., Wang, H., and Li, X. (2019a). Atrasentan Alleviates High Glucose-Induced Podocyte Injury by the microRNA-21/ forkhead Box O1 axis. Eur. J. Pharmacol. 852, 142-150. doi:10.1016/j.ejphar. 2019.03.013

Wang, W., Gao, Q., Yang, M. Y., Zhang, X., Yu, L., Lawas, M., et al. (2015). Upregulation of Lysosomal TRPML1 Channels Is Essential for Lysosomal Adaptation to Nutrient Starvation. Proc. Natl. Acad. Sci. USA 112, E1373-E1381. doi:10.1073/pnas.1419669112

Wang, Y., Huang, H., Jin, Y., Shen, K., Chen, X., Xu, Z., et al. (2019b). Role of TFEB in Autophagic Modulation of Ischemia Reperfusion Injury in Mice Kidney and protection by Urolithin A. Food Chem. Toxicol. 131, 110591. doi:10.1016/j.fct. 2019.110591

Wauson, E. M., Dbouk, H. A., Ghosh, A. B., and Cobb, M. H. (2014). G ProteinCoupled Receptors and the Regulation of Autophagy. Trends Endocrinol. Metab. 25, 274-282. doi:10.1016/j.tem.2014.03.006

Westermark, P., Andersson, A., and Westernark, G. T. (2011). Islet Amyloid Polypeptide, Islet Amyloid, and Diabetes Mellitus. Physiol. Rev. 91, 795-826. doi:10.1152/physrev.00042.2009

White, E. (2012). Deconvoluting the Context-dependent Role for Autophagy in Cancer. Nat. Rev. Cancer 12, 401-410. doi:10.1038/nrc3262

Wild, P., Farhan, H., McEwan, D. G., Wagner, S., Rogov, V. V., Brady, N. R., et al. (2008). Phosphorylation of the Autophagy Receptor Optineurin Restricts Salmonella Growth. Science 333, 228-233. doi:10.1126/science.1205405 
Williams, A., Sarkar, S., Cuddon, P., Ttofi, E. K., Saiki, S., Siddiqi, F. H., et al. (2008). Novel Targets for Huntington's Disease in an mTOR-independent Autophagy Pathway. Nat. Chem. Biol. 4, 295-305. doi:10.1038/nchembio.79

Wu, J., Wu, J. J., Yang, L. J., Wei, L. X., and Zou, D. J. (2013). Rosiglitazone Protects against Palmitate-Induced Pancreatic Beta-Cell Death by Activation of Autophagy via 5'-AMP-Activated Protein Kinase Modulation. Endocrine 44, 87-98. doi:10.1007/s12020-012-9826-5

Xie, Q., Lin, Q., Li, D., and Chen, J. (2017). Imatinib Induced Autophagy via Upregulating XIAP in GIST882 Cells. Biochem. Biophys. Res. Com. 488, 584-589. doi:10.1016/j.bbrc.2017.05.096

Xie, Z., Lau, K., Eby, B., Lozano, P., He, C., Pennington, B., et al. (2011). Improvement of Cardiac Functions by Chronic Metformin Treatment Is Associated with Enhanced Cardiac Autophagy in Diabetic OVE26 Mice. Diabetes 60, 1770-1778. doi:10.2337/db10-0351

Yamada, S., Tanabe, J., Ogura, Y., Nagai, Y., Sugaya, T., Ohata, K., et al. (2021). Renoprotective Effect of GLP-1 Receptor Agonist, Liraglutide, in Early-phase Diabetic Kidney Disease in Spontaneously Diabetic Torii Fatty Rats. Cllin. Exp. Nephrol. 25, 365-375. doi:10.1007/s10157-020-02007-2

Yamamoto, S., Kuramoto, K., Wang, N., Situ, X., Priyadarshini, M., Zhang, W., et al. (2018). Autophagy Differentially Regulates Insulin Production and Insulin Sensitivity. Cel. Rep. 23, 3286-3299. doi:10.1016/j.celrep.2018.05.032

Yan, Y., Wang, Y., Ding, J., Lu, L., Ke, G., and Dong, K. (2021). TRPML1 Inhibited Photoreceptor Apoptosis and Protected the Retina by Activation of Autophagy in Experimental Retinal Detachment. Opthalmic Res. 64, 587-594. doi:10.1159/ 000512104

Yang, L., Li, P., Fu, S., Calay, E. S., and Hotamisligil, G. S. (2010). Defective Hepatic Autophagy in Obesity Promotes ER Stress and Causes Insulin Resistance. Cel. Metab. 11, 467-478. doi:10.1016/j.cmet.2010.04.005

Yang, Y., Fang, F., Xu, G., Zhen, Y., Zhang, Y., Tian, J., et al. (2018). Liraglutide Improves Cognitive Impairment via the AMPK and PI3K/Akt Signaling Pathways in Type 2 Diabetic Rats. Mol. Med. Rep. 18, 2449-2457. doi:10. 3892/mmr.2018.9180

Yusta, B., Baggio, L. L., Estall, J. L., Koehler, J. A., Holland, D. P., Li, H., et al. (2006). GLP-1 Receptor Activation Improves Beta Cell Function and Survival Following Induction of Endoplasmic Reticulum Stress. Cel. Metab. 4, 391-406. doi:10.1016/j.cmet.2006.10.001

Zhang, F., Liu, K., Cao, M., Qu, J., Zhou, D., Pan, Z., et al. (2019a). Rosiglitazone Treatment Prevents Postoperative Fibrosis in a Rabbit Model of Glaucoma Filtration Surgery. Invest. Opthalmol. Vis. Sci. 60, 2473-2452. doi:10.1167/iovs. $18-26526$

Zhang, H., Alsaleh, G., Feltham, J., Sun, Y., Napolitano, G., Riffelmacher, T., et al. (2019b). Polyamines Control eIF5A Hypusination, TFEB Translation, and Autophagy to Reverse B Cell Senescence. Mol. Cell 79, 110-125. doi:10. 1016/j.molcel.2019.08.005

Zhang, J., Cheng, Y., Gu, J., Wang, S., Zhou, S., Wang, Y., et al. (2016). Fenofibrate Increases Cardiac Autophagy via FGF21/SIRT1 and Prevents Fibrosis and Inflammation in the Hearts of Type 1 Diabetic Mice. Clin. Sci. 130, 625-641. doi:10.1042/cs20150623

Zhang, L., Yu, J., Pan, H., Hu, P., Hao, Y., Cai, W., et al. (2007). Small Molecule Regulators of Autophagy Identified by an Image-Based High-Throughput Screen. Proc. Natl. Acad. Sci. USA 104, 190223-119028. doi:10.1073/pnas. 0709695104

Zhang, L., Zhang, L., Li, Y., Li, L., Melchiorsen, J. U., Rosenkilde, M., et al. (2020a). The Novel Dual GLP-1/GIP Receptor Agonist DA-CH5 Is Superior to Single
GLP-1 Receptor Agonists in the MPTP Model of Parkinson's Disease. J. Parkinsons Dis. 10, 523-542. doi:10.3233/jpd-191768

Zhang, Y., Goldman, S., Baerga, R., Zhao, Y., Komatsu, M., and Jin, S. (2009). Adipose-specific Deletion of Autophagy-Related Gene 7 (Atg7) in Mice Reveals a Role in Adipogenesis. Proc. Natl. Acad. Sci. USA 106, 19860-19865. doi:10. 1073/pnas.0906048106

Zhang, Y., Shaikh, N., Ferey, J. L., Wankhade, U. D., Chintapalli, S. V., Higgins, C. B., et al. (2020b). Lactotrehalose, an Analog of Trehalose, Increases Energy Metabolism without Promoting Clostridioides Difficile Infection in Mice. Gastroenterology 158, 1402-1416. doi:10.1053/j.gastro.2019.11.295

Zhang, Y., Yang, Y., Yu, H., Li, M., Hang, L., and Xu, X. (2020c). Apigenin Protects Mouse Retina against Oxidative Damage by Regulating the Nrf2 Pathway and Autophagy. Oxid. Med. Cel. Longev. 2020, 9420704. doi:10. $1155 / 2020 / 9420704$

Zhao, Y., Zhang, W., Jia, Q., Feng, Z., Guo, J., Han, X., et al. (2019). High Dose Vitamin E Attenuates Diabetic Nephropathy via Alleviation of Autophagic Stress. Front. Physiol. 9, 1939. doi:10.3389/fphys.2018.01939

Zheng, H. J., Zhang, X., Guo, J., Zhang, W., Ai, S., Zhang, F., et al. (2020). Lysosomal Dysfunction-Induced Autophagic Stress in Diabetic Kidney Disease. J. Cel. Mol. Med. 24, 8276-8290. doi:10.1111/jcmm.15301

Zheng, R. H., Zhang, W. W., Ji, Y. N., Bai, X. J., Yan, C. P., Wang, J., et al. (2019). Exogenous Supplement of Glucagon like Peptide-1 Protects the Heart against Aortic Banding Induced Myocardial Fibrosis and Dysfunction through Inhibiting mTOR/p70S6K Signaling and Promoting Autophagy. Eur. J. Pharmacol. 883, 173318. doi:10.1016/j.ejphar.2020.173318

Zheng, Y., Kou, J., Wang, P., Ye, T., Wang, Z., Gao, Z., et al. (2021). Berberineinduced TFEB Deacetylation by SIRT1 Promotes Autophagy in Peritoneal Macrophages. Aging 13, 7096-7119. doi:10.18632/aging.202566

Zhou, G., Myers, R., Li, Y., Chen, Y., Shen, X., Fenyk-Melody, J., et al. (2003). Role of AMP-Activated Protein Kinase in Mechanism of Metformin Action. J. Clin. Invest. 108, 1167-1174. doi:10.1172/JCI13505

Zhuang, J., Ji, X., Zhu, Y., Liu, W., Sun, J., Jiao, X., et al. (2020). Restriction of Intracellular Salmonella typhimurium Growth by the Small-Molecule Autophagy Inducer A77 1726 through the Activation of the AMPK-ULK1 axis. Vet. Microbiol. 254, 108982.

Conflict of Interest: M-SL is the CEO of LysoTech, Corp.

The remaining author declares that the research was conducted in the absence of any commercial or financial relationships that could be construed as a potential conflict of interest.

Publisher's Note: All claims expressed in this article are solely those of the authors and do not necessarily represent those of their affiliated organizations, or those of the publisher, the editors and the reviewers. Any product that may be evaluated in this article, or claim that may be made by its manufacturer, is not guaranteed or endorsed by the publisher.

Copyright (C) 2022 Park and Lee. This is an open-access article distributed under the terms of the Creative Commons Attribution License (CC BY). The use, distribution or reproduction in other forums is permitted, provided the original author(s) and the copyright owner(s) are credited and that the original publication in this journal is cited, in accordance with accepted academic practice. No use, distribution or reproduction is permitted which does not comply with these terms. 